\title{
Are the eigenvalues of preconditioned banded symmetric Toeplitz matrices known in almost closed form?
}

\author{
Fayyaz Ahmad' ${ }^{1}$ Eman Salem Al-Aidarous ${ }^{2}$. \\ Dina Abdullah Alrehaili ${ }^{2}$ Sven-Erik Ekström ${ }^{3}$ (D) \\ Isabella Furci $^{1}$ - Stefano Serra-Capizzano ${ }^{1,3}$
}

Received: 28 June 2017 / Accepted: 15 August 2017 / Published online: 31 August 2017 (C) The Author(s) 2017. This article is an open access publication

\begin{abstract}
Bogoya, Böttcher, Grudsky, and Maximenko have recently obtained the precise asymptotic expansion for the eigenvalues of a sequence of Toeplitz matrices $\left\{T_{n}(f)\right\}$, under suitable assumptions on the associated generating function $f$. In this paper, we provide numerical evidence that some of these assumptions can be relaxed and extended to the case of a sequence of preconditioned Toeplitz matrices $\left\{T_{n}^{-1}(g) T_{n}(f)\right\}$, for $f$ trigonometric polynomial, $g$ nonnegative, not identically zero trigonometric polynomial, $r=f / g$, and where the ratio $r$ plays the same role as $f$
\end{abstract}

Sven-Erik Ekström

sven-erik.ekstrom@it.uu.se

Fayyaz Ahmad

fahmad@uninsubria.it

Eman Salem Al-Aidarous

ealaidarous@kau.edu.sa

Dina Abdullah Alrehaili

dalrehaili@stu.kau.edu.sa

Isabella Furci

ifurci@uninsubria.it

Stefano Serra-Capizzano

stefano.serrac@uninsubria.it; stefano.serra@it.uu.se

1 Department of Science and High Technology, University of Insubria, Via Valleggio 11, 22100 Como, Italy

2 Faculty of Science, Department of Mathematics, King Abdulaziz University, P.O. Box 80203, 21589 Jeddah, Saudi Arabia

3 Division of Scientific Computing, Department of Information Technology, ITC, Uppsala University, Lägerhyddsv. 2, Hus 2, P.O. Box 337, 75105 Uppsala, Sweden 
in the nonpreconditioned case. Moreover, based on the eigenvalue asymptotics, we devise an extrapolation algorithm for computing the eigenvalues of preconditioned banded symmetric Toeplitz matrices with a high level of accuracy, with a relatively low computational cost, and with potential application to the computation of the spectrum of differential operators.

Keywords (Preconditioned) Toeplitz matrix · Mass and stiffness matrix ·

Eigenvalues · Eigenvalue asymptotics $\cdot$ Polynomial interpolation $\cdot$ Extrapolation

Mathematics Subject Classifications (2010) $15 \mathrm{~B} 05 \cdot 65 \mathrm{~F} 15 \cdot 65 \mathrm{D} 05 \cdot 65 \mathrm{~B} 05$

\section{Introduction}

A matrix of size $n$, having a fixed entry along each diagonal, is called Toeplitz and enjoys the expression

$$
\left[a_{i-j}\right]_{i, j=1}^{n}=\left[\begin{array}{cccccc}
a_{0} & a_{-1} & a_{-2} & \cdots & \cdots & a_{-(n-1)} \\
a_{1} & \ddots & \ddots & \ddots & & \vdots \\
a_{2} & \ddots & \ddots & \ddots & \ddots & \vdots \\
\vdots & \ddots & \ddots & \ddots & \ddots & a_{-2} \\
\vdots & & \ddots & \ddots & \ddots & a_{-1} \\
a_{n-1} & \cdots & \cdots & a_{2} & a_{1} & a_{0}
\end{array}\right] .
$$

Given a complex-valued Lebesgue integrable function $\phi:[-\pi, \pi] \rightarrow \mathbb{C}$, the $n$th Toeplitz matrix generated by $\phi$ is defined as

$$
T_{n}(\phi)=\left[\hat{\phi}_{i-j}\right]_{i, j=1}^{n},
$$

where the quantities $\hat{\phi}_{k}$ are the Fourier coefficients of $\phi$, which means

$$
\hat{\phi}_{k}=\frac{1}{2 \pi} \int_{-\pi}^{\pi} \phi(\theta) \mathrm{e}^{-\mathrm{i} k \theta} \mathrm{d} \theta, \quad k \in \mathbb{Z} .
$$

We refer to $\left\{T_{n}(\phi)\right\}_{n}$ as the Toeplitz sequence generated by $\phi$, which in turn is called the generating function of $\left\{T_{n}(\phi)\right\}_{n}$. In the case where $\phi$ is real-valued, all the matrices $T_{n}(\phi)$ are Hermitian and much is known about their spectral properties, from the localization of the eigenvalues to the asymptotic spectral distribution in the Weyl sense: in particular $\phi$ is the spectral symbol of $\left\{T_{n}(\phi)\right\}_{n}$, see [7,14] and the references therein.

More in detail, if $\phi$ is real-valued and not identically constant, then any eigenvalue of $T_{n}(\phi)$ belongs to the open set $\left(m_{\phi}, M_{\phi}\right)$, with $m_{\phi}, M_{\phi}$ being the essential infimum, the essential supremum of $\phi$, respectively. The case of a constant $\phi$ is trivial: in that case if $\phi=m$ almost everywhere then $T_{n}(\phi)=m \mathbb{I}_{n}$ with $\mathbb{I}_{n}$ denoting the identity of size $n$. Hence if $M_{\phi}>0$ and $\phi$ is nonnegative almost everywhere, then $T_{n}(\phi)$ is Hermitian positive definite.

In this paper, we focus our attention on the following setting. 
- We consider two real-valued cosine trigonometric polynomials (RCTPs) $f, g$, that is

$$
\begin{array}{lll}
f(\theta)=\hat{f}_{0}+2 \sum_{k=1}^{m_{1}} \hat{f}_{k} \cos (k \theta), & \hat{f}_{0}, \hat{f}_{1}, \ldots, \hat{f}_{m_{1}} \in \mathbb{R}, & m_{1} \in \mathbb{N}, \\
g(\theta)=\hat{g}_{0}+2 \sum_{k=1}^{m_{2}} \hat{g}_{k} \cos (k \theta), & \hat{g}_{0}, \hat{g}_{1}, \ldots, \hat{g}_{m_{2}} \in \mathbb{R}, \quad m_{2} \in \mathbb{N},
\end{array}
$$

so that $T_{n}(f), T_{n}(g)$ are both real symmetric.

- We assume that $M_{g}=\max g>0$ and $m_{g}=\min g \geq 0$, so that $T_{n}(g)$ is positive definite.

- We consider $\mathcal{P}_{n}(f, g)=T_{n}^{-1}(g) T_{n}(f)$ the "preconditioned" matrix and we define the new symbol $r=f / g$.

The $n$th Toeplitz matrix generated by $\phi \in\{f, g\}$ is the real symmetric banded matrix of bandwidth $2 m+1, m \in\left\{m_{1}, m_{2}\right\}\left(m=m_{1}\right.$ if $\phi=f$ and $m=m_{2}$ if $\phi=g$ ), given by

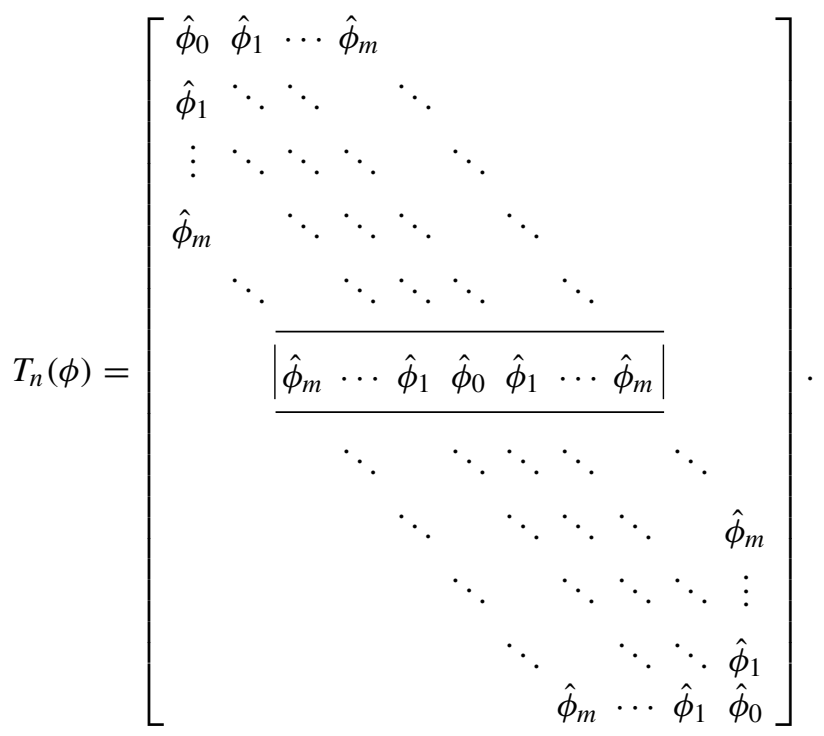

Matrices of the form $\mathcal{P}_{n}(f, g)$ are important for the fast solution of large Toeplitz linear systems (in connection with the preconditioned conjugate gradient method [911, 18] or of more general preconditioned Krylov methods [15, 16]). Furthermore, up to low rank corrections, they appear in the context of the spectral approximation of differential operators in which a low rank correction of $T_{n}(g)$ is the mass matrix and a low rank correction of $T_{n}(f)$ is the stiffness matrix.

Their spectral features have been studied in detail. More precisely, under the assumption that $r=m$ identically $\mathcal{P}_{n}(f, g)=r \mathbb{I}_{n}$, while if $m_{r}<M_{r}$, then any eigenvalue of $\mathcal{P}_{n}(f, g)$ belongs to the open set $\left(m_{r}, M_{r}\right)$, see [11], and the whole sequence $\left\{\mathcal{P}_{n}(f, g)\right\}_{n}$ is spectrally distributed in the Weyl sense as $r=f / g$ (see [19]). 
In our context, we say that a function is monotone if it is either increasing or decreasing over the interval $[0, \pi]$.

Under the assumption that $r=f / g$ is monotone, in this paper, we show experimentally that for every integer $\alpha \geq 0$, every $n$ and every $j=1, \ldots, n$, the following asymptotic expansion holds:

$$
\lambda_{j}\left(\mathcal{P}_{n}(f, g)\right)=r\left(\theta_{j, n}\right)+\sum_{k=1}^{\alpha} c_{k}\left(\theta_{j, n}\right) h^{k}+E_{j, n, \alpha},
$$

where:

- the eigenvalues of $\mathcal{P}_{n}(f, g)$ are arranged in nondecreasing or nonincreasing order, depending on whether $r$ is increasing or decreasing;

- $\left\{c_{k}\right\}_{k=1,2, \ldots}$ is a sequence of functions from $[0, \pi]$ to $\mathbb{R}$ which depends only on $r$;

- $h=\frac{1}{n+1}$ and $\theta_{j, n}=\frac{j \pi}{n+1}=j \pi h$;

- $E_{j, n, \alpha}=O\left(h^{\alpha+1}\right)$ is the remainder (the error), which satisfies the inequality $\left|E_{j, n, \alpha}\right| \leq C_{\alpha} h^{\alpha+1}$ for some constant $C_{\alpha}$ depending only on $\alpha$ and $r$.

In the pure Toeplitz case, that is for $g=1$ identically, so that $\mathcal{P}_{n}(f, g)=T_{n}(f)$ and $r=f$, the result is proven in [4-6], if the RCTP $f$ is monotone and satisfies certain additional assumptions, which include the requirements that $f^{\prime}(\theta) \neq 0$ for $\theta \in(0, \pi)$ and $f^{\prime \prime}(\theta) \neq 0$ for $\theta \in\{0, \pi\}$. The symbols

$$
f_{q}(\theta)=(2-2 \cos \theta)^{q}, \quad q=1,2, \ldots,
$$

arise in the discretization of differential equations and are therefore of particular interest. Unfortunately, for these symbols, the requirement that $f^{\prime \prime}(0) \neq 0$ is not satisfied if $q \geq 2$. In [13], several numerical evidences are reported, showing that the higher order approximation (1) holds even in this "degenerate case."

Here, as first purpose, we show numerically the same for the preconditioned matrices $\mathcal{P}_{n}(f, g)$ and, from a theoretical point of view, the numerical testing is complemented by the proof of the above conjecture in the basic case of $\alpha=0$.

Furthermore, in [13], the authors employed the asymptotic expansion (1) for computing an accurate approximation of $\lambda_{j}\left(T_{n}(f)\right)$ for very large $n$, provided that the values $\lambda_{j_{1}}\left(T_{n_{1}}(f)\right), \ldots, \lambda_{j_{s}}\left(T_{n_{s}}(f)\right)$ are available for moderate sizes $n_{1}, \ldots, n_{s}$ with $\theta_{j_{1}, n_{1}}=\cdots=\theta_{j_{s}, n_{s}}=\theta_{j, n}, s \geq 2$. The second and main purpose of this paper is to carry out this idea and to support it by numerical experiments, accompanied by an appropriate error analysis in the more general case of the preconditioned matrices $\mathcal{P}_{n}(f, g)$. In particular, we devise an algorithm to compute $\lambda_{j}\left(\mathcal{P}_{n}(f, g)\right)$ with a high level of accuracy and a relatively low computational cost. The algorithm is completely analogous to the extrapolation procedure, which is employed in the context of Romberg integration (to obtain high precision approximations of an integral from a few coarse trapezoidal approximations [20, Section 3.4], see also [8] for more advanced algorithms). In this regard, the asymptotic expansion (1) plays here the same role as the Euler-Maclaurin summation formula [20, Section 3.3].

The third and last purpose of this paper is to formulate, on the basis of numerical experiments, a conjecture on the higher-order asymptotic of the eigenvalues if the monotonicity assumption on $r=f / g$ is not in force. We also illustrate how this 
conjecture can be used along with our extrapolation algorithm in order to compute some of the eigenvalues of $\mathcal{P}_{n}(f, g)$ in the case where $r$ is nonmonotone.

\section{Error bounds for the coefficients $c_{k}$ in the asymptotic expansion}

We start this section by manipulating the error expression implicitly given in (1), the goal being that of using extrapolation methods [8]. In fact, if we assume that the relations in (1) hold, then we can write

$$
E_{j, n, 0}=\sum_{k=1}^{\alpha} c_{k}\left(\theta_{j, n}\right) h^{k}+E_{j, n, \alpha},
$$

where $E_{j, n, 0}=\lambda_{j}\left(\mathcal{P}_{n}(f, g)\right)-r\left(\theta_{j, n}\right)$.

We now suppose to know the eigenvalues for different (small) $n_{i}$ namely $\left\{\left(n_{1}, \lambda_{j_{1}}\left(\mathcal{P}_{n_{1}}(f, g)\right)\right), \quad\left(n_{2}, \lambda_{j_{2}}\left(\mathcal{P}_{n_{2}}(f, g)\right)\right), \cdots, \quad\left(n_{\alpha}, \lambda_{j_{\alpha}}\left(\mathcal{P}_{n_{\alpha}}(f, g)\right)\right)\right\}$, where $n_{1}, n_{2}, \cdots, n_{\alpha}$ and $j_{1}, j_{2}, \cdots, j_{\alpha}$ are chosen in such a way that $j_{1} /\left(n_{1}+1\right)=j_{2} /\left(n_{2}+1\right)=\cdots=j_{\alpha} /\left(n_{\alpha}+1\right)$.

By defining $h_{1}=1 /\left(n_{1}+1\right), h_{2}=1 /\left(n_{2}+1\right), \ldots, h_{\alpha}=1 /\left(n_{\alpha}+1\right)$, for a given set of eigenvalues, (3) can be written as

$$
\begin{gathered}
E_{j_{1}, n_{1}, 0}=\sum_{k=1}^{\alpha} c_{k}\left(\theta_{j_{1}, n_{1}}\right) h_{1}^{k}+E_{j_{1}, n_{1}, \alpha}, \\
E_{j_{2}, n_{2}, 0}=\sum_{k=1}^{\alpha} c_{k}\left(\theta_{j_{2}, n_{2}}\right) h_{2}^{k}+E_{j_{2}, n_{2}, \alpha}, \\
E_{j_{3}, n_{3}, 0}=\sum_{k=1}^{\alpha} c_{k}\left(\theta_{j_{3}, n_{3}}\right) h_{3}^{k}+E_{j_{3}, n_{3}, \alpha}, \\
\vdots \\
E_{j_{\alpha}, n_{\alpha}, 0}=\sum_{k=1}^{\alpha} c_{k}\left(\theta_{j_{\alpha}, n_{\alpha}}\right) h_{\alpha}^{k}+E_{j_{\alpha}, n_{\alpha}, \alpha} .
\end{gathered}
$$

Let $c, \tilde{c}$ be the vectors

$$
c=\left[c_{1}, c_{2}, \ldots, c_{\alpha}\right]^{T} ; \quad \tilde{c}=\left[\tilde{c}_{1}, \tilde{c}_{2}, \ldots, \tilde{c}_{\alpha}\right]^{T},
$$

and let $A$ be the coefficient matrix of size $\alpha \times \alpha$ with $(A)_{i, j}=h_{i}^{j}$. Hence, the set of (4) can be written in matrix form as

$$
A c=b_{0}-b_{\alpha},
$$

where $b_{0}=\left[E_{j_{1}, n_{1}, 0}, E_{j_{2}, n_{2}, 0}, \ldots, E_{j_{\alpha}, n_{\alpha}, 0}\right]^{T}$ and $b_{\alpha}=$ $\left[E_{j_{1}, n_{1}, \alpha}, E_{j_{2}, n_{2}, \alpha}, \ldots, E_{j_{\alpha}, n_{\alpha}, \alpha}\right]^{T}$. Furthermore, by neglecting the higher order errors, we may define an approximation $\tilde{c}$ of $c$ according to the expression below

$$
A \tilde{c}=b_{0} .
$$

By solving the linear system of equations above, the approximation of $c$ is easily obtained since the matrix size is very small. In a subsequent step, we derive upperbounds for $|\tilde{c}-c|$ : in reality, (5) and (6) leads to

$$
A(\tilde{c}-c)=b_{\alpha} .
$$


If we define $\Delta c=\tilde{c}-c$ and $\eta_{i}=\frac{E_{j_{i}, n_{i}, \alpha}}{h_{i}^{\alpha+1}}$ for $i=1, \ldots, \alpha$, then the system (7) can be written as

$$
A \Delta c=\left[\begin{array}{c}
\eta_{1} h_{1}^{\alpha+1} \\
\eta_{2} h_{2}^{\alpha+1} \\
\vdots \\
\eta_{\alpha} h_{\alpha}^{\alpha+1}
\end{array}\right]
$$

with $\left|\eta_{i}\right| \leq C_{\alpha}$ for $i=1, \ldots, \alpha$, where $C_{\alpha}$ is a constant. The coefficient matrix can be expressed as

$$
A=\left[\begin{array}{cccc}
h_{1} & h_{1}^{2} & \ldots & h_{1}^{\alpha} \\
h_{2} & h_{2}^{2} & \ldots & h_{2}^{\alpha} \\
\vdots & \vdots & & \vdots \\
h_{\alpha} & h_{\alpha}^{2} & \ldots & h_{\alpha}^{\alpha}
\end{array}\right]=\left[\begin{array}{cccc}
h_{1} & & & \\
& h_{2} & & \\
& & \ddots & \\
& & & h_{\alpha}
\end{array}\right] V\left(h_{1}, \ldots, h_{\alpha}\right),
$$

where $V\left(h_{1}, \ldots, h_{\alpha}\right)$ is the Vandermonde matrix of order $\alpha$ corresponding to $h_{1}, \ldots, h_{\alpha}$.

By assuming $W=V^{-1}\left(h_{1}, \ldots, h_{\alpha}\right)$, we deduce

$$
(W)_{i, j}=\left\{\begin{array}{c}
\sum_{\substack{1 \leq k_{1}<\ldots<k_{\alpha-i} \leq \alpha \\
k_{1}, \ldots, k_{\alpha-i} \neq j}} h_{k_{1}} \cdots h_{k_{\alpha-i}} \\
\prod_{\substack{1 \leq k \leq \alpha \\
k \neq j}}\left(h_{j}-h_{k}\right) \\
\prod_{\substack{1 \leq k \leq \alpha \\
k \neq j}}\left(h_{j}-h_{k}\right)
\end{array} \quad i=\alpha .\right.
$$

Therefore for the inversion of the matrix $A$, we have

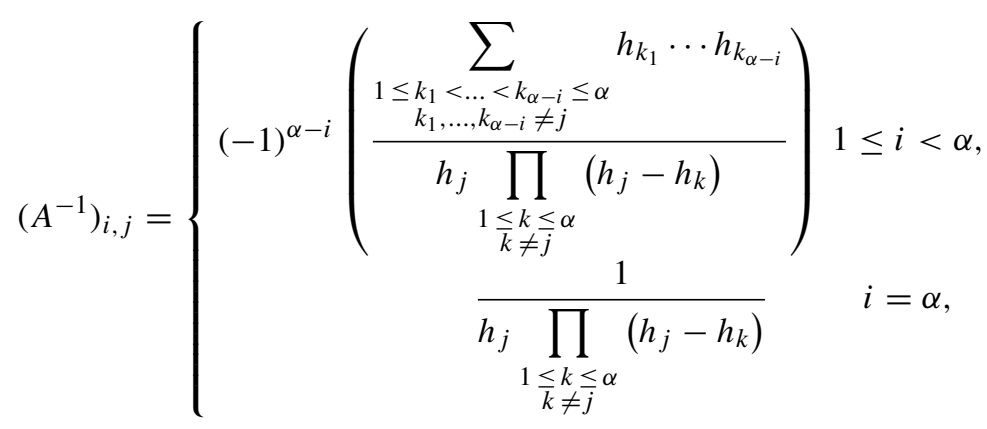

and we can obtain an explicit expression for $(\Delta c)_{i}, i=1, \ldots, \alpha$, that is

$$
(\Delta c)_{i}=\sum_{j=1}^{\alpha}\left(A^{-1}\right)_{i, j} \eta_{j} h_{j}^{\alpha+1} .
$$


Case 1 If $i=\alpha$, then

$$
(\Delta c)_{\alpha}=\sum_{j=1}^{\alpha} \frac{\eta_{j} h_{j}^{\alpha+1}}{h_{j} \prod_{\substack{1 \leq k \leq \alpha \\ k \neq j}}\left(h_{j}-h_{k}\right)}
$$

Whence, from the fact that $\left|\eta_{i}\right| \leq C_{\alpha}$ for $i=1, \ldots, \alpha$,

$$
\left|(\Delta c)_{\alpha}\right| \leq \sum_{j=1}^{\alpha} \frac{\left|\eta_{j}\right| h_{j}^{\alpha+1}}{h_{j} \prod_{\substack{1 \leq k \leq \alpha \\ k \neq j}}\left|h_{j}-h_{k}\right|} \leq \sum_{j=1}^{\alpha} \frac{C_{\alpha} h_{j}^{\alpha}}{\prod_{\substack{1 \leq k \leq \alpha \\ k \neq j}}\left|h_{j}-h_{k}\right|}
$$

With the choice $h_{j}=\frac{1}{m^{j-1}} h_{1}$ for $j=1, \ldots, \alpha, m$ positive integer, we have

$$
\begin{gathered}
\left|(\Delta c)_{\alpha}\right| \leq C_{\alpha} \sum_{j=1}^{\alpha} \frac{\left(\frac{h_{1}}{m^{j-1}}\right)^{\alpha}}{\prod_{\substack{1 \leq k \leq \alpha \\
k \neq j}} h_{1}\left|\frac{1}{m^{j-1}}-\frac{1}{m^{k-1}}\right|}=C_{\alpha} h_{1}^{\alpha} \sum_{j=1}^{\alpha} \frac{\left(\frac{1}{m^{j-1}}\right)^{\alpha}}{h_{1}^{\alpha-1} \prod_{\substack{1 \leq k \leq \alpha \\
k \neq j}}\left|\frac{1}{m^{j-1}}-\frac{1}{m^{k-1}}\right|} \\
=h_{1} C_{\alpha} \sum_{j=1}^{\alpha} \frac{\left(\frac{1}{m^{j-1}}\right)^{\alpha}}{\prod_{\substack{1 \leq k \leq \alpha \\
k \neq j}}\left|\frac{1}{m^{j-1}}-\frac{1}{m^{k-1}}\right|}=O\left(h_{1}\right) .
\end{gathered}
$$

Case 2 If $i=1, \ldots, \alpha-1$, then

$$
(\Delta c)_{i}=\sum_{j=1}^{\alpha}(-1)^{\alpha-i} \eta_{j} h_{j}^{\alpha+1} \frac{\sum_{\substack{1 \leq k_{1}<\ldots<k_{\alpha-i} \leq \alpha \\ k_{1}, \ldots, k_{\alpha-i} \neq j}} h_{k_{1}} \cdots h_{k_{\alpha-i}}}{h_{j} \prod_{\substack{1 \leq k \leq \alpha \\ k \neq j}}\left(h_{j}-h_{k}\right)},
$$

that is different from the case $i=\alpha$ just for the numerator

$$
\sum_{\substack{1 \leq k_{1}<\ldots<k_{\alpha-i} \leq \alpha \\ k_{1}, \ldots, k_{\alpha-i} \neq j}} h_{k_{1}} \cdots h_{k_{\alpha-i}} .
$$

As a consequence,

$$
\left|(\Delta c)_{i}\right| \leq C_{\alpha} \sum_{j=1}^{\alpha} h_{j}^{\alpha} \frac{\sum_{\substack{1 \leq k_{1}<\ldots<k_{\alpha-i} \leq \alpha \\ k_{1}, \ldots, k_{\alpha-i} \neq j}} h_{k_{1}} \cdots h_{k_{\alpha-i}}}{\prod_{\substack{1 \leq k \leq \alpha \\ k \neq j}}\left|h_{j}-h_{k}\right|} .
$$


With the choice $h_{j}=\frac{1}{m^{j-1}} h_{1}$ for $j=1, \ldots, \alpha$, we infer

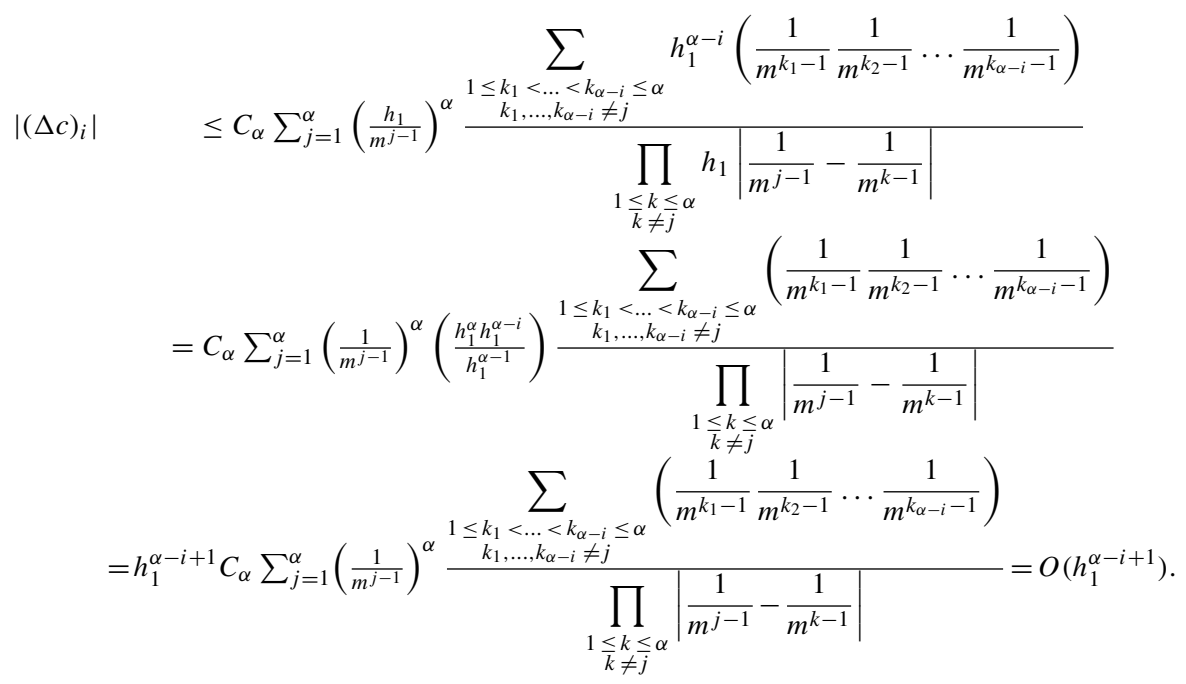

As a conclusion, with the choice $h_{j}=\frac{1}{m^{j-1}} h_{1}$ for $j=1, \ldots, \alpha$ and under the assumption that the asymptotic expansion reported in (1) is true, we deduce

$$
\left|(\Delta c)_{i}\right|=O\left(h_{1}^{\alpha-i+1}\right)
$$

for $i=1, \ldots, \alpha$.

\section{Error bounds for numerically approximated eigenvalues}

The goal of this short section is to provide error bounds based on the linear system in (6) for the computation of the eigenvalues of $\mathcal{P}_{n}(f, g)$ : of course, these error bounds are based on the conjecture that the relations reported in (1) are true. However, as we can see in Section 4, the numerical tests fully support the existence of the considered asymptotic expansion.

Indeed, as already observed, by solving (6), we can approximate $c_{k}$. Once we have the values of $c_{k}$, we can approximate the eigenvalues $\lambda_{j_{\beta}}$ of a large dimension matrix of size $n_{\beta}$, here $n_{\beta}+1=m^{\beta-1}\left(n_{1}+1\right)$. The asymptotic expansion (3) can be written as

$$
E_{j_{\beta}, n_{\beta}, 0}=\bar{h}_{\beta}^{T} c+E_{j_{\beta}, n_{\beta}, \alpha} .
$$

By subtraction $\bar{h}_{\beta}^{T} \tilde{c}$ from both sides of the equation above, we find

$$
\begin{aligned}
& E_{j_{\beta}, n_{\beta}, 0}-\bar{h}_{\beta}^{T} \tilde{c} \quad=\bar{h}_{\beta}^{T}(c-\tilde{c})+E_{j_{\beta}, n_{\beta}, \alpha}, \\
& \lambda_{j}\left(\mathcal{P}_{n_{\beta}}(f, g)\right)-r\left(\theta_{j, n_{\beta}}\right)-\bar{h}_{\beta}^{T} \tilde{c} \quad=\bar{h}_{\beta}^{T} \Delta c+E_{j_{\beta}, n_{\beta}, \alpha}, \\
& \left|\lambda_{j}\left(\mathcal{P}_{n_{\beta}}(f, g)\right)-r\left(\theta_{j, n_{\beta}}\right)-\bar{h}_{\beta}^{T} \tilde{c}\right| \leq \sum_{i=1}^{\alpha} h_{\beta}^{i}\left|(\Delta c)_{i}\right|+\left|E_{j_{\beta}, n_{\beta}, \alpha}\right|, \\
& \left|\lambda_{j}\left(\mathcal{P}_{n_{\beta}}(f, g)\right)-r\left(\theta_{j, n_{\beta}}\right)-\bar{h}_{\beta}^{T} \tilde{c}\right| \leq \sum_{i=1}^{\alpha} h_{\beta}^{i}\left|(\Delta c)_{i}\right|+C_{\alpha} h_{\beta}^{\alpha+1},
\end{aligned}
$$


where $\bar{h}_{\beta}=\left[h_{\beta}, h_{\beta}^{2}, \cdots, h_{\beta}^{\alpha}\right]^{T},\left|E_{j_{\beta}, n_{\beta}, \alpha}\right| \leq C_{\alpha} h_{\beta}^{\alpha+1}$ for some constant $C_{\alpha}$ and $\left|(\Delta c)_{i}\right|$ is given in (12).

\section{Numerical tests}

In this section, we want to present a few numerical experiments to support the asymptotic expansion (1) in the case where one or more properties of the following list are satisfied:

1. $f^{\prime \prime}(0) \neq 0$ (see Example 1, Example 3, and Example 5),

2. $f^{\prime \prime}(0)=0$ (see Example 2 and Example 4),

3. $\min g>0$ (see Example 1, Example 2, and Example 5),

4. $\min g=0$ (see Example 3 and Example 4),

5. $r=f / g$ is non monotone (see Example 5).

The approximation of eigenvalues of large matrices in each case is also computed. The expansion (1) for $\alpha=4$ is

$$
\begin{aligned}
\lambda_{j}\left(\mathcal{P}_{n}(f, g)\right)= & r\left(\theta_{j, n}\right)+c_{1}\left(\theta_{j, n}\right) h+c_{2}\left(\theta_{j, n}\right) h^{2}+c_{3}\left(\theta_{j, n}\right) h^{3}+c_{4}\left(\theta_{j, n}\right) h^{4}+E_{j, n, 4}, \\
E_{j, n, 0}= & \lambda_{j}\left(\mathcal{P}_{n}(f, g)\right)-r\left(\theta_{j, n}\right)=c_{1}\left(\theta_{j, n}\right) h+c_{2}\left(\theta_{j, n}\right) h^{2}+c_{3}\left(\theta_{j, n}\right) h^{3} \\
& +c_{4}\left(\theta_{j, n}\right) h^{4}+E_{j, n, 4} .
\end{aligned}
$$

In all numerical examples, we choose four matrix-size values, that is $n_{i}$ for $i \in$ $\{1,2,3,4\}$, in a way that they satisfy $n_{i}=m^{i-1}\left(n_{1}+1\right)-1$, with $m$ being a positive integer. The expansion (15) for the set of the four dimensions $n_{i}$ can be written as

$$
\begin{aligned}
& E_{j_{1}, n_{1}, 0}=c_{1}\left(\theta_{j_{1}, n_{1}}\right) h_{1}+c_{2}\left(\theta_{j_{1}, n_{1}}\right) h_{1}^{2}+c_{3}\left(\theta_{j_{1}, n_{1}}\right) h_{1}^{3}+c_{4}\left(\theta_{j_{1}, n_{1}}\right) h_{1}^{4}+E_{j_{1}, n_{1}, 4}, \\
& E_{j_{2}, n_{2}, 0}=c_{1}\left(\theta_{j_{2}, n_{2}}\right) h_{2}+c_{2}\left(\theta_{j_{2}, n_{2}}\right) h_{2}^{2}+c_{3}\left(\theta_{j_{2}, n_{2}}\right) h_{2}^{3}+c_{4}\left(\theta_{j_{2}, n_{2}}\right) h_{2}^{4}+E_{j_{2}, n_{2}, 4}, \\
& E_{j_{3}, n_{3}, 0}=c_{1}\left(\theta_{j_{3}, n_{3}}\right) h_{3}+c_{2}\left(\theta_{j_{3}, n_{3}}\right) h_{3}^{2}+c_{3}\left(\theta_{j_{3}, n_{3}}\right) h_{3}^{3}+c_{4}\left(\theta_{j_{3}, n_{3}}\right) h_{3}^{4}+E_{j_{3}, n_{3}, 4}, \\
& E_{j_{4}, n_{4}, 0}=c_{1}\left(\theta_{j_{4}, n_{4}}\right) h_{4}+c_{2}\left(\theta_{j_{4}, n_{4}}\right) h_{4}^{2}+c_{3}\left(\theta_{j_{4}, n_{4}}\right) h_{4}^{3}+c_{4}\left(\theta_{j_{4}, n_{4}}\right) h_{4}^{4}+E_{j_{4}, n_{4}, 4}^{4},
\end{aligned}
$$

where $h_{i}=\frac{1}{n_{i}+1}$ and $j_{i}=m^{i-1} j_{1}$ for $i \in\{1,2,3,4\}$. Notice that $\theta_{j_{i}, n_{i}}=\theta_{j_{1}, n_{1}}=\bar{\theta}$ for a fixed $j_{1} \in\left\{1,2, \cdots, n_{1}\right\}$. We are interested in the numerical approximation of $c_{i}(\bar{\theta})$ for $i \in\{1,2,3,4\}$ and then in the precise numerical approximation of the eigenvalue of $\mathcal{P}_{n}(f, g)$ for large $n$. The set of (16) can be written as

$$
\begin{aligned}
& E_{j_{1}, n_{1}, 0}=\tilde{c}_{1}(\bar{\theta}) h_{1}+\tilde{c}_{2}(\bar{\theta}) h_{1}^{2}+\tilde{c}_{3}(\bar{\theta}) h_{1}^{3}+\tilde{c}_{4}(\bar{\theta}) h_{1}^{4}, \\
& E_{j_{2}, n_{2}, 0}=\tilde{c}_{1}(\bar{\theta}) h_{2}+\tilde{c}_{2}(\bar{\theta}) h_{2}^{2}+\tilde{c}_{3}(\bar{\theta}) h_{2}^{3}+\tilde{c}_{4}(\bar{\theta}) h_{2}^{4}, \\
& E_{j_{3}, n_{3}, 0}=\tilde{c}_{1}(\bar{\theta}) h_{3}+\tilde{c}_{2}(\bar{\theta}) h_{3}^{2}+\tilde{c}_{3}(\bar{\theta}) h_{3}^{3}+\tilde{c}_{4}(\bar{\theta}) h_{3}^{4}, \\
& E_{j_{4}, n_{4}, 0}=\tilde{c}_{1}(\bar{\theta}) h_{4}+\tilde{c}_{2}(\bar{\theta}) h_{4}^{2}+\tilde{c}_{3}(\bar{\theta}) h_{4}^{3}+\tilde{c}_{4}(\bar{\theta}) h_{4}^{4} .
\end{aligned}
$$

We solve the system of linear equations above for $j_{1} \in\left\{1,2, \cdots, n_{1}\right\}$ to compute $\tilde{c}_{i}(\bar{\theta})$. The computed $\tilde{c}_{i}$ are used to approximate the eigenvalues of large size $n_{\beta}$ by exploiting the following relation

$$
\tilde{\lambda}_{j_{\beta}}\left(\mathcal{P}_{n_{\beta}}(f, g)\right)=r\left(\theta_{j_{\beta}, n_{\beta}}\right)+\bar{h}_{\beta}^{T} \tilde{c} .
$$


Example 1 Let $g, f$, and $r$ be the functions defined as

$$
\begin{aligned}
& f(\theta)=4-2 \cos (\theta)-2 \cos (2 \theta)=(2-2 \cos (\theta))(3+2 \cos (\theta)), \\
& g(\theta)=3+2 \cos (\theta), \\
& r(\theta)=\frac{f(\theta)}{g(\theta)}=2-2 \cos (\theta),
\end{aligned}
$$

where $\theta \in[0, \pi]$. The graphs of generating functions are shown in left panel of Fig. 1, and the approximations $\tilde{c}_{k}$, for $k=1,2,3,4$ are shown in the right panel. Note that $g(\theta)>0, \forall \theta \in[0, \pi], f^{\prime \prime}(0) \neq 0$, and furthermore $r$ is monotone. We set $n=n_{1} \in\{40,60,80,100\}$ and $m=2$.

Example 2 Let $g, f$, and $r$ be the functions defined as

$$
\begin{aligned}
& f(\theta)=20-30 \cos (\theta)+12 \cos (2 \theta)-2 \cos (3 \theta)=(2-2 \cos (\theta))^{3}, \\
& g(\theta)=3+2 \cos (\theta), \\
& r(\theta)=\frac{f(\theta)}{g(\theta)}=\frac{(2-2 \cos (\theta))^{3}}{3+2 \cos (\theta)},
\end{aligned}
$$

where $\theta \in[0, \pi]$. The graphs of generating functions are shown in left panel of Fig. 2, and the approximations $\tilde{c}_{k}$, for $k=1,2,3,4$ are shown in the right panel. Remark that $g(\theta)>0, \forall \theta \in[0, \pi], f^{\prime \prime}(0)=0$, and furthermore $r$ is monotone. We set $n=n_{1} \in\{40,60,80,100\}$ and $m=2$.

There is an important issue to discuss here. Both the functions $f$ and $r$ attain the minimum at $\theta=0$ with a very high order. Indeed, we have $f(\theta), r(\theta) \approx \theta^{6}$, with $\phi_{1} \approx \phi_{2}$ being the symmetric, transitive relation telling that there exist positive constants $c, C>0$ such that $c \phi_{1} \leq \phi_{2} \leq C \phi_{1}$ on the whole definition domain $[0, \pi]$. Therefore for fixed $j$ (independent of $n$ ) the $j$ th smallest eigenvalue of $\mathcal{P}_{n}(f, g)$ is asymptotic to $k_{j} h^{6}, k_{j}$ positive constant depending on $j$ but not on $n$ : the reader is refereed to [17] for the preconditioned case with the limitation $j=1$ and to [1] and references therein for very elegant and precise estimates regarding the pure Toeplitz case.
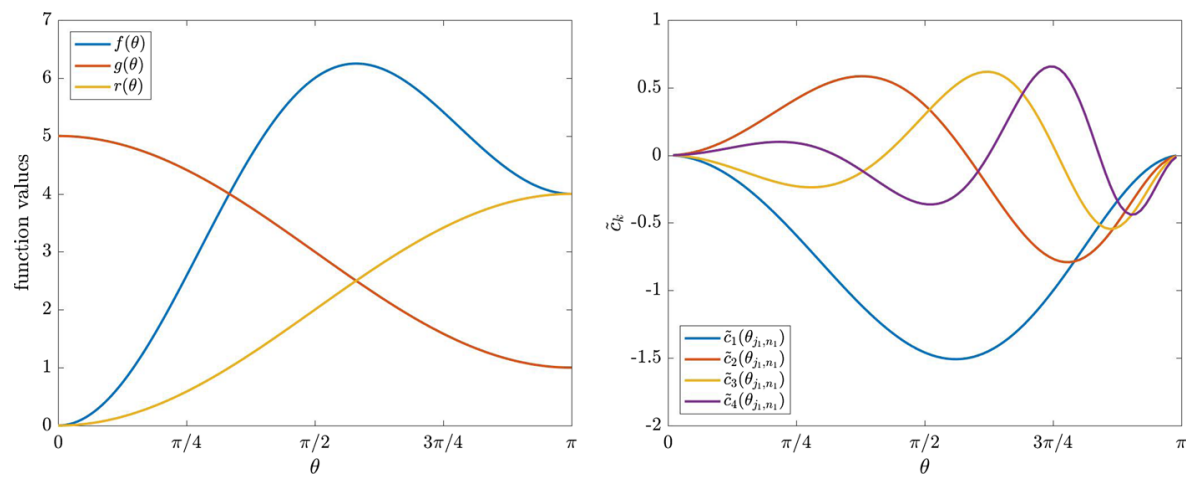

Fig. 1 Example 1: Generating functions $(f, g$, and $r)$ and $\tilde{c}_{k}$ for $k=1,2,3,4$ 

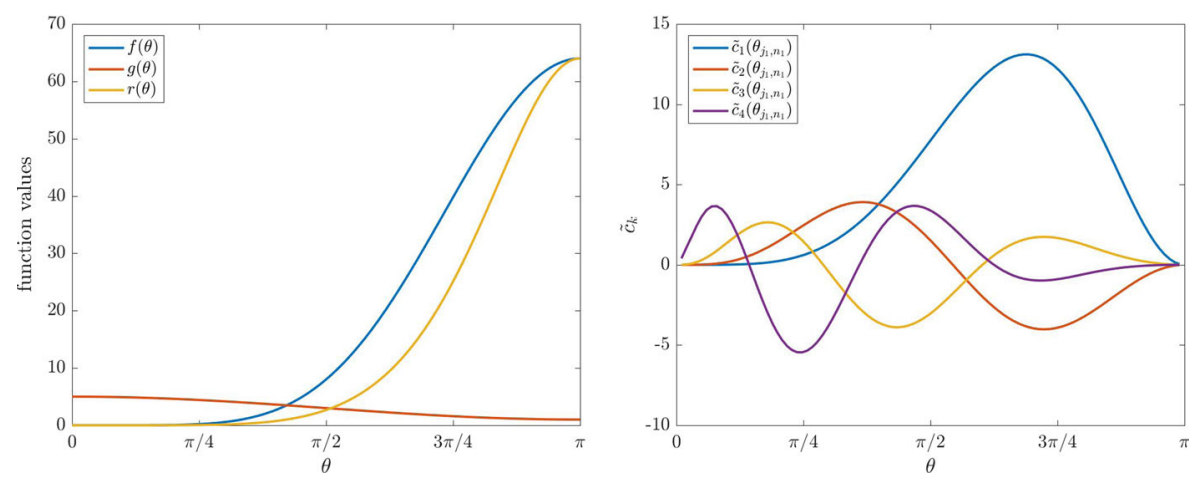

Fig. 2 Example 2: generating functions $(f, g$, and $r)$ and $\tilde{c}_{k}$ for $k=1,2,3,4$

Now if we fix $j$ and we put together $\lambda_{j}\left(\mathcal{P}_{n}(f, g)\right) \approx h^{6}$ with relations (3)(4), then the only possibility for avoiding a contradiction is that the functions $c_{1}(\theta), c_{2}(\theta), c_{3}(\theta), c_{4}(\theta), c_{5}(\theta)$ all vanish at $\theta=0$.

The approximations $\tilde{c}_{k}$, for $k=1,2,3,4$ shown in the right panel of Fig. 2 are coherent with the above mathematical conclusion and in fact all these approximations vanish simultaneously at $\theta=0$ (the fifth is not displayed, but we computed it and it also equals to zero at $\theta=0$, while, as expected from an extension of the results by [1] to the preconditioned Toeplitz case, the sixth is nonzero at $\theta=0$ ).

Since the argument and the conclusions are the very same, we anticipate that the discussion can be repeated verbatim for Example 4, where the functions $f$ and $r$ attain the minimum at $\theta=0$ with order 10 . As a consequence, we expect that the functions $c_{1}(\theta), \ldots, c_{9}(\theta)$ all simultaneously vanish at $\theta=0$, while $c_{10}(0) \neq$ 0 : this is confirmed for the first four of them as reported in the right panel of Fig. 4.
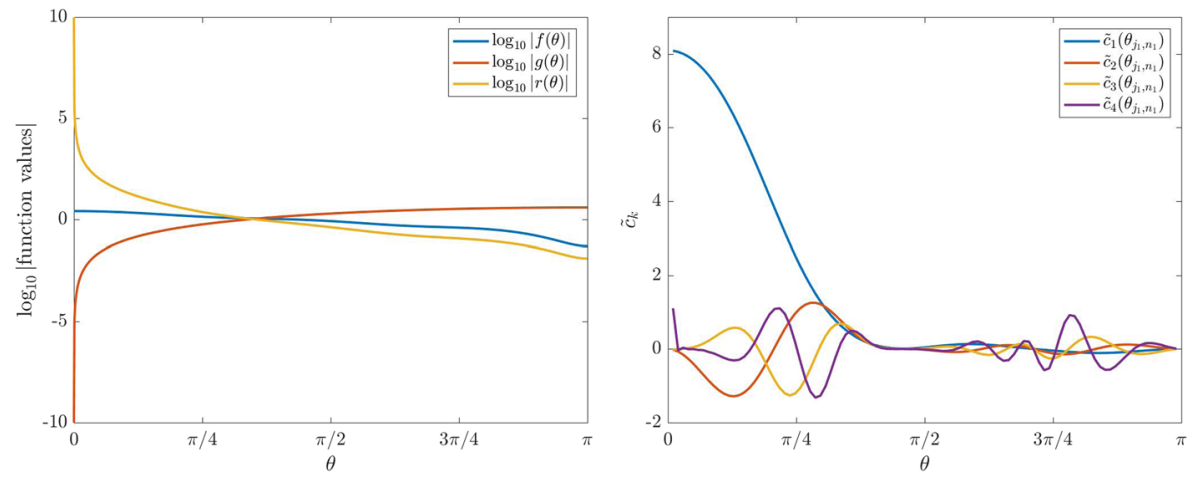

Fig. 3 Example 3: generating functions $(f, g$, and $r)$ and $\tilde{c}_{k}$ for $k=1,2,3,4$ 

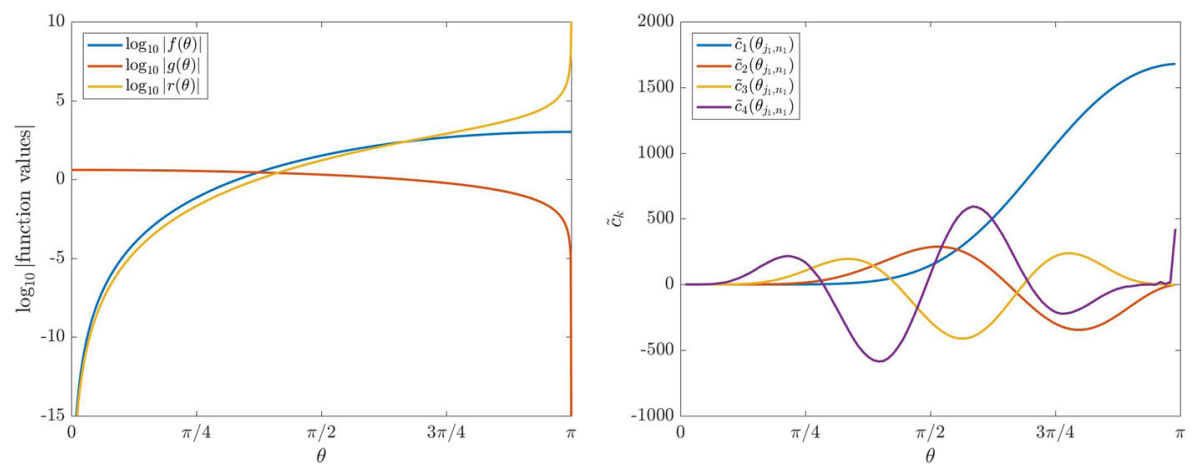

Fig. 4 Example 4: generating functions $(f, g$, and $r)$ and $\tilde{c}_{k}$ for $k=1,2,3,4$

Example 3 Let $g, f$, and $r$ be the functions defined as

$$
\begin{aligned}
& f(\theta)=1+\cos (\theta)+\frac{1}{4} \cos (2 \theta)+\frac{1}{5} \cos (3 \theta)+\frac{1}{10} \cos (4 \theta)+\frac{1}{10} \cos (5 \theta), \\
& g(\theta)=2-2 \cos (\theta), \\
& r(\theta)=\frac{f(\theta)}{g(\theta)}=\frac{1+\cos (\theta)+\frac{1}{4} \cos (2 \theta)+\frac{1}{5} \cos (3 \theta)+\frac{1}{10} \cos (4 \theta)+\frac{1}{10} \cos (5 \theta)}{2-2 \cos (\theta)},
\end{aligned}
$$

where $\theta \in[0, \pi]$. The graphs of generating functions are shown in left panel of Fig. 3, and the approximations $\tilde{c}_{k}$, for $k=1,2,3,4$ are shown in the right panel. Note that $\min g(\theta)=0, \forall \theta \in[0, \pi], f^{\prime \prime}(0) \neq 0$, and furthermore $r$ is monotone. We set $n=n_{1} \in\{40,60,80,100\}$ and $m=2$.

Example 4 Let $g, f$, and $r$ be the functions defined as

$$
\begin{aligned}
& f(\theta)=252-420 \cos (\theta)+240 \cos (2 \theta)-90 \cos (3 \theta)+20 \cos (4 \theta)-2 \cos (5 \theta)=(2-2 \cos (\theta))^{5}, \\
& g(\theta)=2+2 \cos (\theta), \\
& r(\theta)=\frac{f(\theta)}{g(\theta)}=\frac{(2-2 \cos (\theta))^{5}}{2+2 \cos (\theta)},
\end{aligned}
$$
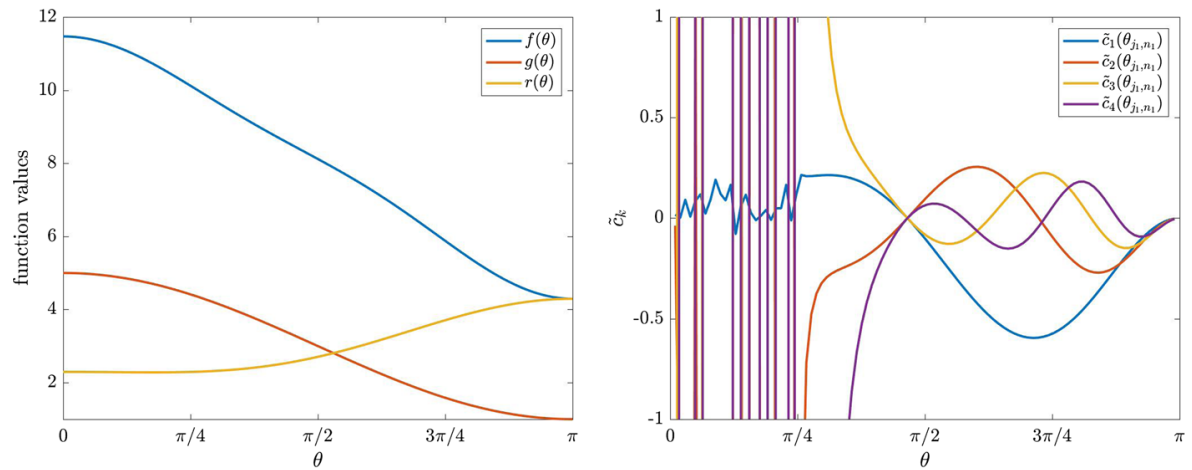

Fig. 5 Example 5: generating functions $(f, g$, and $r)$ and $\tilde{c}_{k}$ for $k=1,2,3,4$ 

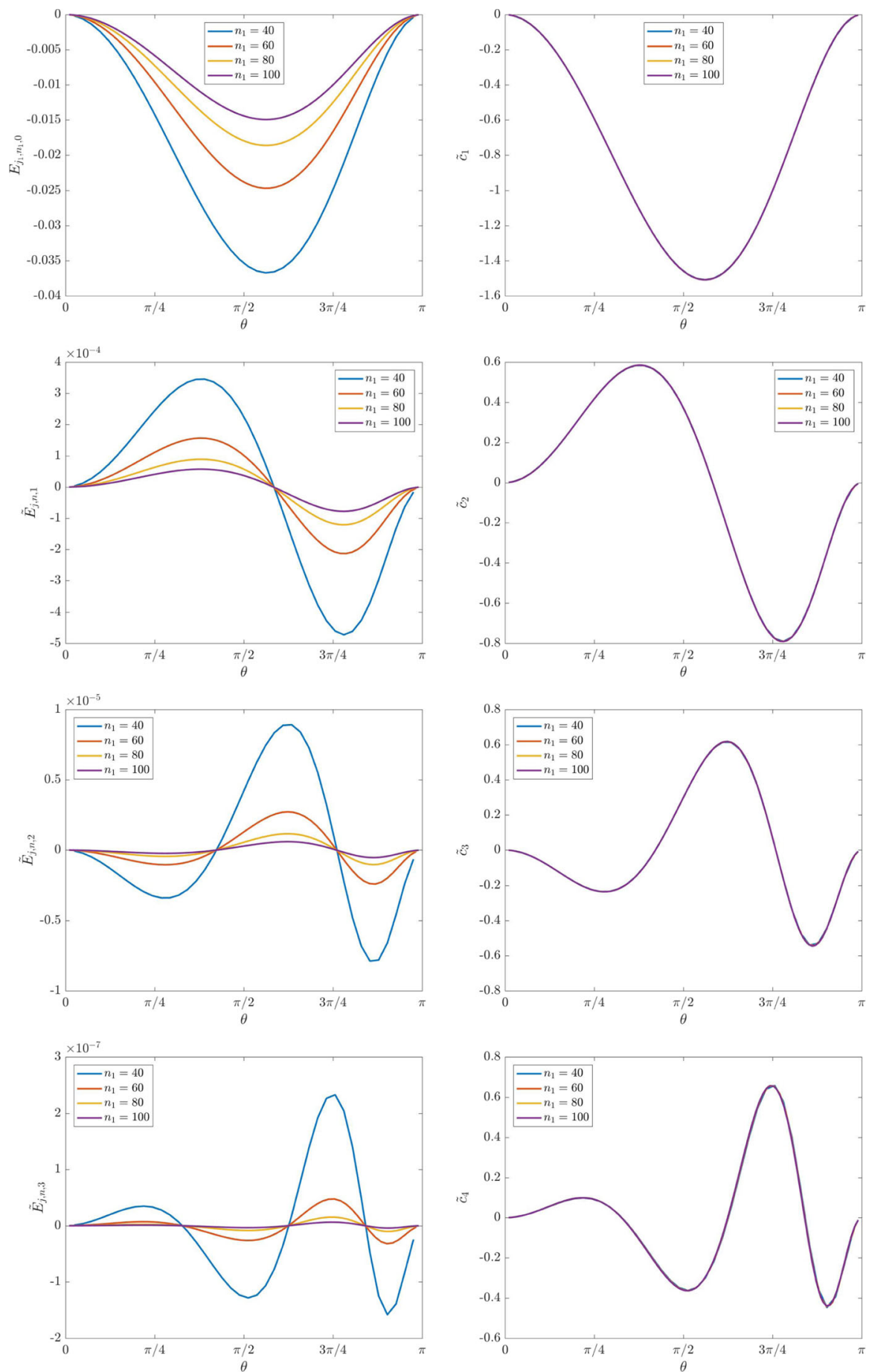

Fig. 6 Example 1: $E_{j, n, 0}, \tilde{E}_{j, n, k}(k=1,2,3)$, and $\tilde{c}_{k}(k=1,2,3,4)$, for $n=n_{1}=\{40,60,80,100\}$ 

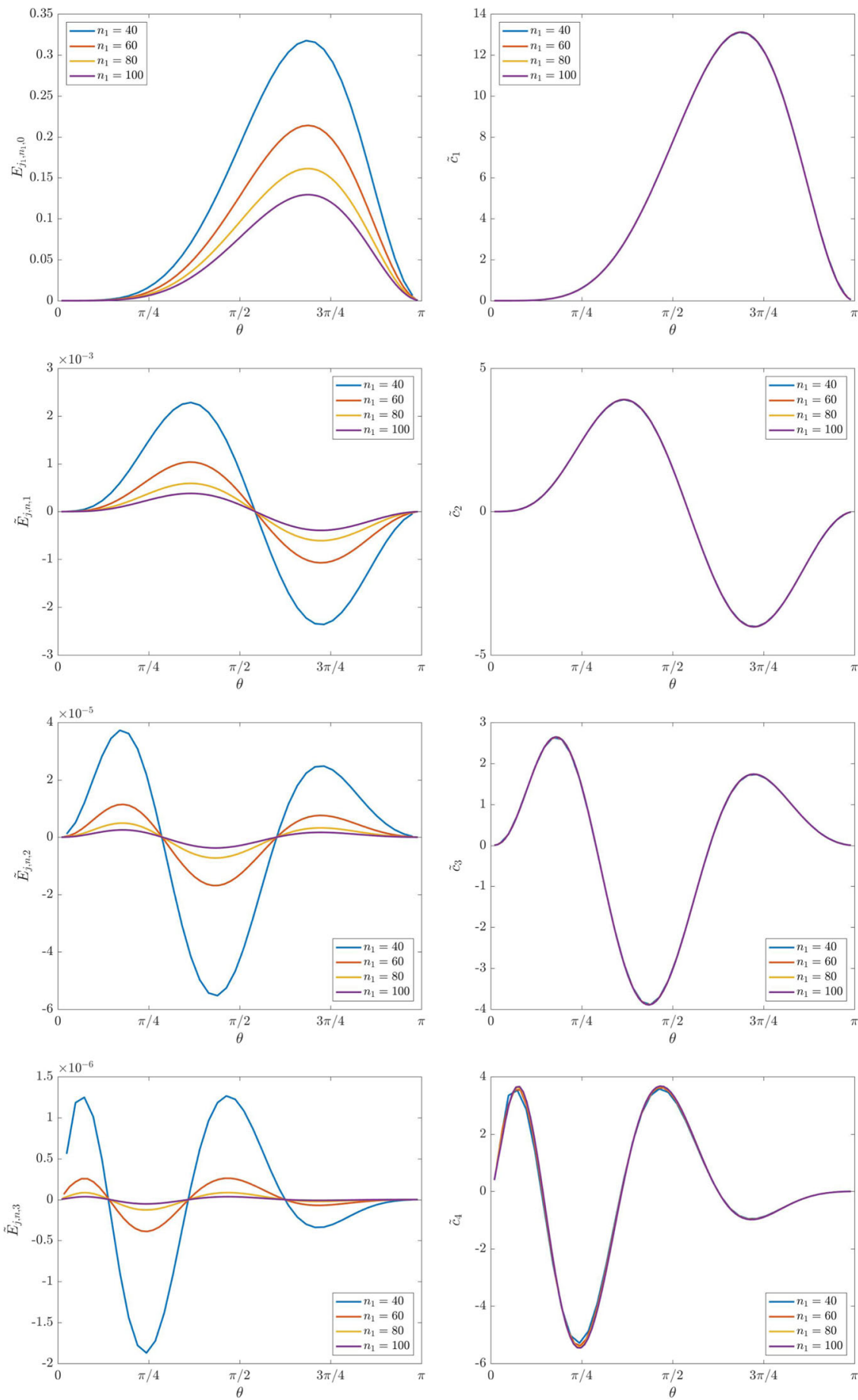

Fig. 7 Example 2: $E_{j, n, 0}, \tilde{E}_{j, n, k}(k=1,2,3)$, and $\tilde{c}_{k}(k=1,2,3,4)$, for $n=n_{1}=\{40,60,80,100\}$ 

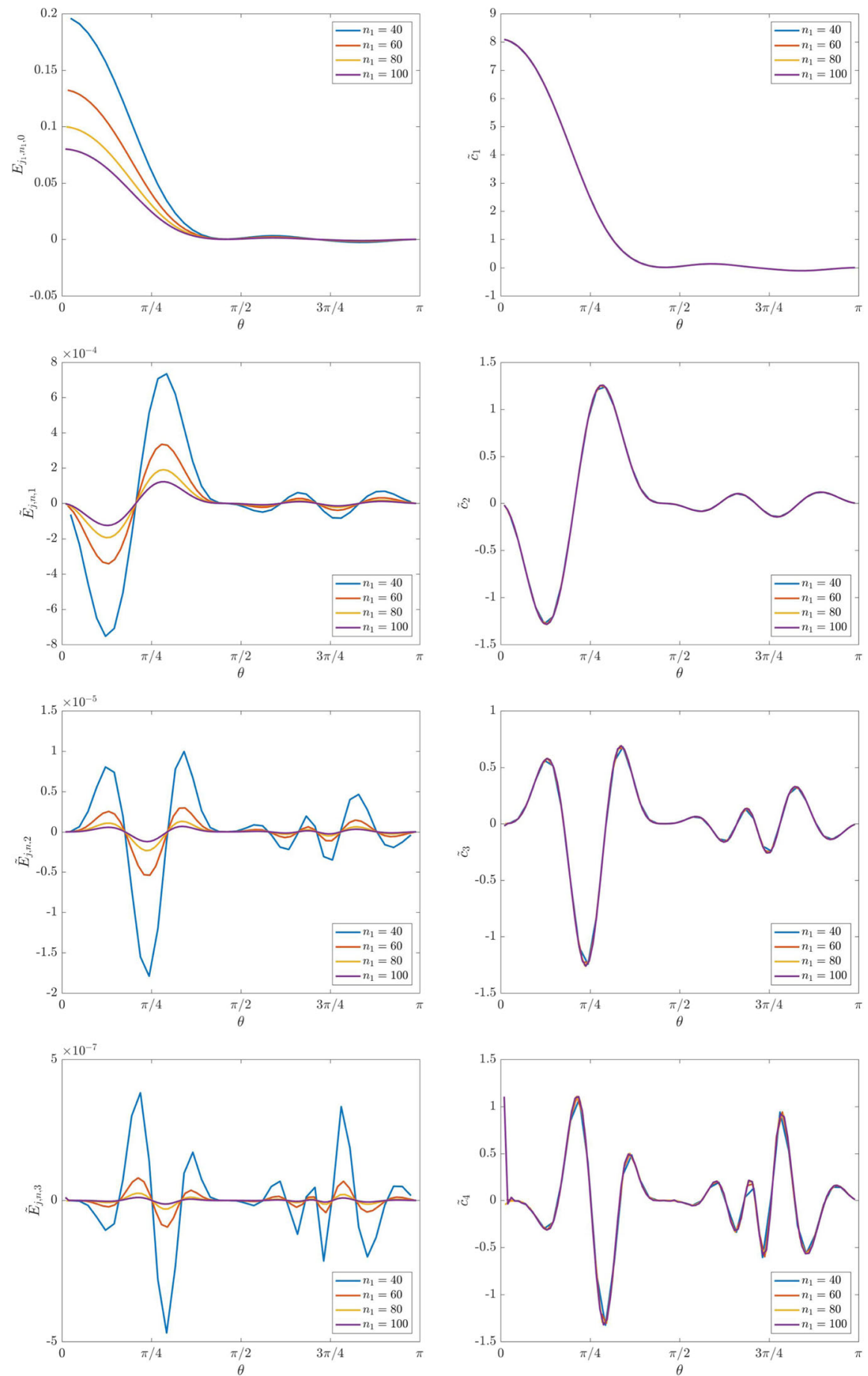

Fig. 8 Example 3: $E_{j, n, 0}, \tilde{E}_{j, n, k}(k=1,2,3)$, and $\tilde{c}_{k}(k=1,2,3,4)$, for $n=n_{1}=\{40,60,80,100\}$ 

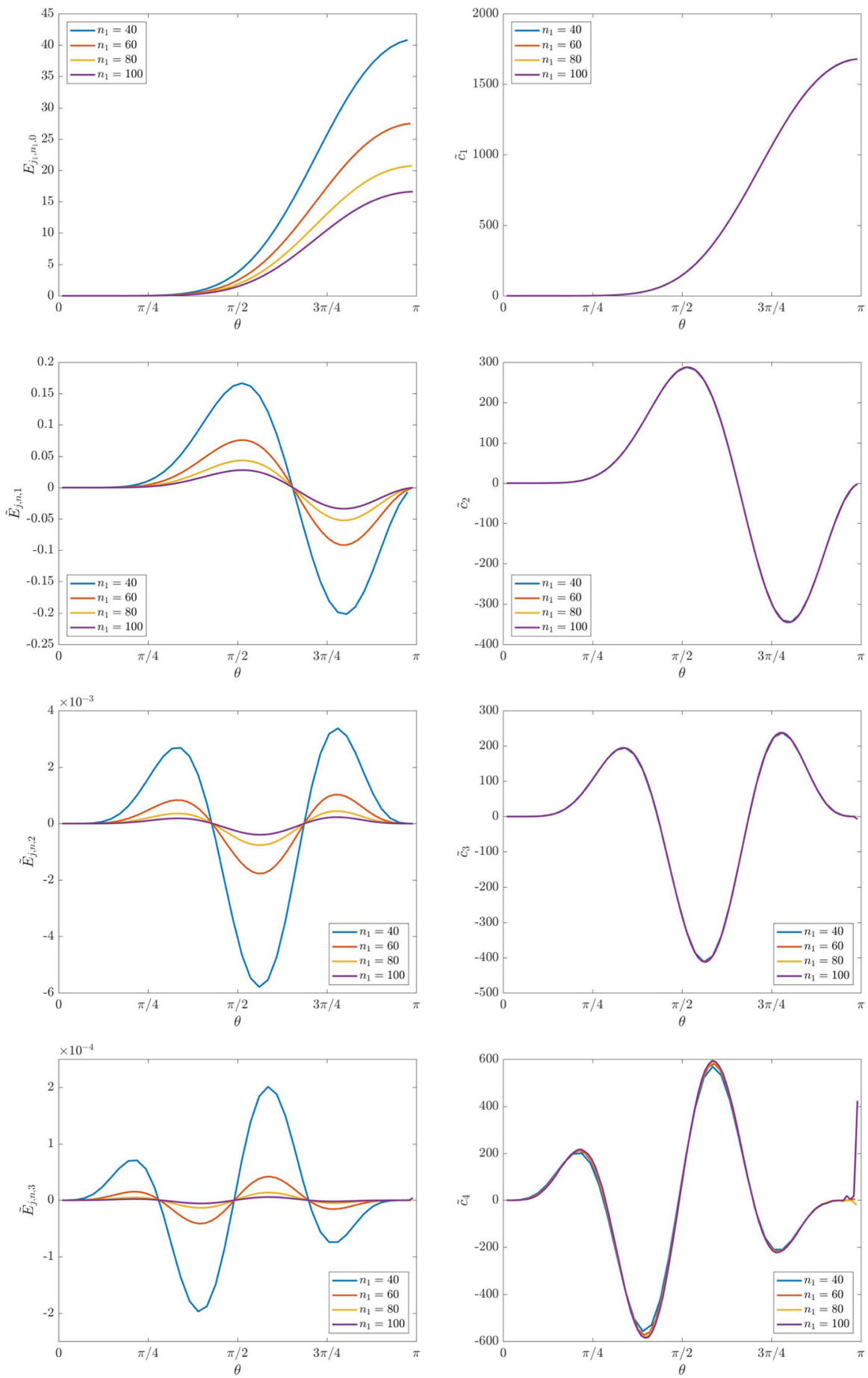

Fig. 9 Example 4: $E_{j, n, 0}, \tilde{E}_{j, n, k}(k=1,2,3)$, and $\tilde{c}_{k}(k=1,2,3,4)$, for $n=n_{1}=\{40,60,80,100\}$ 

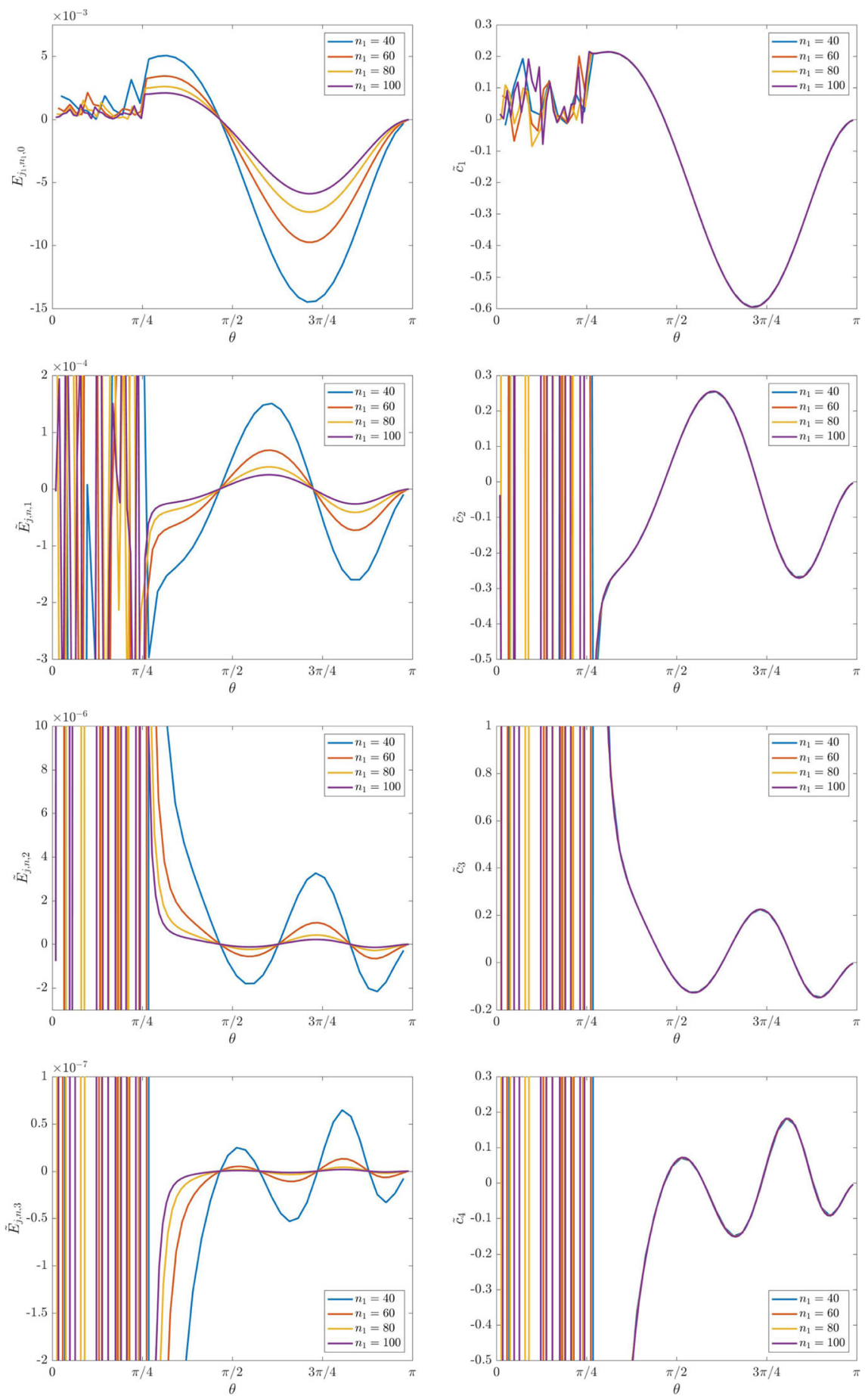

Fig. 10 Example 5: $E_{j, n, 0}, \tilde{E}_{j, n, k}(k=1,2,3)$, and $\tilde{c}_{k}(k=1,2,3,4)$, for $n=n_{1}=\{40,60,80,100\}$ 
where $\theta \in[0, \pi]$. The graphs of generating functions are shown in left panel of Fig. 4, and the approximations $\tilde{c}_{k}$, for $k=1,2,3,4$ are shown in the right panel. Remark that $\min g(\theta)=0, \forall \theta \in[0, \pi], f^{\prime \prime}(0)=0$, and furthermore $r$ is monotone. We set $n=n_{1} \in\{40,60,80,100\}$ and $m=2$.

Example 5 Let $g, f$, and $r$ be the functions defined as

$$
\begin{aligned}
& f(\theta)=\frac{136}{17}+\frac{56}{17} \cos (\theta)-\frac{2}{17} \cos (2 \theta)+\frac{5}{17} \cos (3 \theta)=\left(3-\cos (\theta)+\frac{5}{17} \cos (2 \theta)\right)(3+2 \cos (\theta)), \\
& g(\theta)=3+2 \cos (\theta), \\
& r(\theta)=\frac{f(\theta)}{g(\theta)}=3-\cos (\theta)+\frac{5}{17} \cos (2 \theta),
\end{aligned}
$$

where $\theta \in[0, \pi]$. The graphs of generating functions are shown in left panel of Fig. 5, and the approximations $\tilde{c}_{k}$, for $k=1,2,3,4$ are shown in the right panel. Notice that $\min g(\theta)>0, \forall \theta \in[0, \pi], f^{\prime \prime}(0) \neq 0$, and furthermore $r$ is non monotone. We set $n=n_{1} \in\{40,60,80,100\}$ and $m=2$.

The numerical tests related to Examples 1 and 2, as in Figs. 6 and 7, show that the error expansion (1) behaves as expected. In Fig. 11, we also see that the approximated $\tilde{c}_{k}$ can be used for a large $n$ to approximate the error term to (or almost to) machine precision.

In the numerical tests associated with Examples 3 and 4, as in Figs. 8 and 9, we observe again that the error expansion is in accordance with (1). We also note a slight deviation for the largest eigenvalue and this has to be expected since we have $r\left(\theta_{1, n}\right) \rightarrow \infty$ as $n \rightarrow \infty$ for Example 3 (on the other hand for Example 4 we notice $r\left(\theta_{n, n}\right) \rightarrow \infty$ as $\left.n \rightarrow \infty\right)$. However, the approximation of the eigenvalues of $\mathcal{P}_{n}(f, g)$ is excellent and almost to machine precision as reported in Fig. 12.

In the numerical test related to Example 5, we have a non monotone region for $\theta \in\left[0,2 \tan ^{-1}(\sqrt{3 / 17})\right]$ where the proposed expansion does not work. Indeed, additional errors are introduced when compared to $E_{j, n, 0}$, since the sampling of $r\left(\theta_{j_{1}, n_{1}}\right)$ leads to a poorer approximation after ordering than the procedure given by sampling $r\left(\theta_{j, n_{7}}\right)$ first and then picking samples after ordering. However, the expansion is
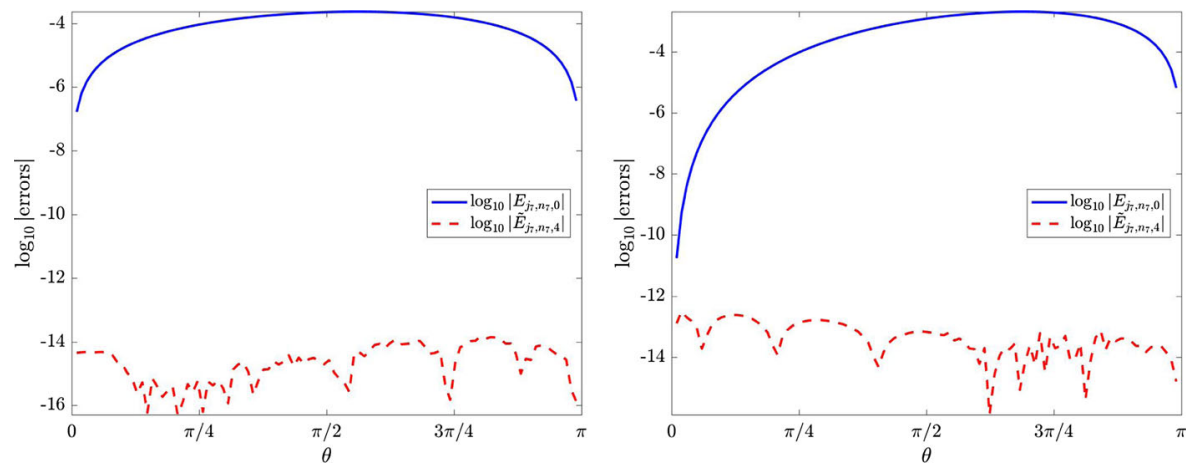

Fig. 11 Example 1 and 2: the errors $\log _{10}\left|E_{j_{7}, n_{7}, 0}\right|$ and $\log _{10}\left|\tilde{E}_{j_{7}, n_{7}, 4}\right|$ for the 100 indices $j_{7}$ of $n_{7}=$ 6463 in (18), corresponding to $n_{1}=100$, and using $\tilde{c}_{k}, k=1,2,3,4$, computed with $m=2$ 

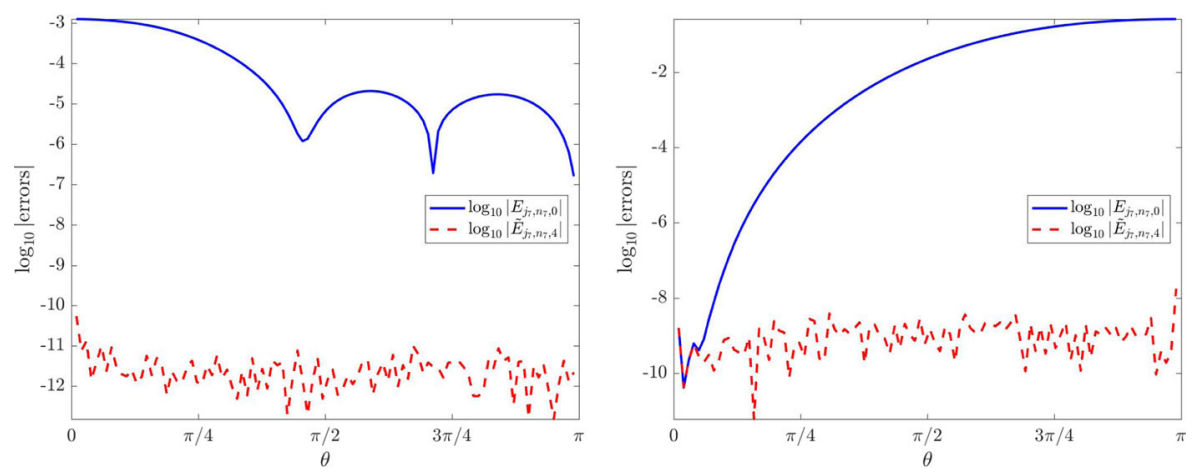

Fig. 12 Example 3 and 4: the errors $\log _{10}\left|E_{j_{7}, n_{7}, 0}\right|$ and $\log _{10}\left|\tilde{E}_{j 7, n_{7}, 4}\right|$ for the 100 indices $j_{7}$ of $n_{7}=$ 6463 in (18), corresponding to $n_{1}=100$, and using $\tilde{c}_{k}, k=1,2,3$, 4, computed with $m=2$

confirmed for the rest of the domain, as seen in Fig. 10. Furthermore, in Fig. 13, the expansion works well again for the monotone part, by allowing an approximation almost to machine precision of the eigenvalues of $\mathcal{P}_{n}(f, g)$.

However, even if the eigenvalues lying in the non monotone region give raise to an irregular error pattern, it seems that there exists a kind of 'deformed' periodicity in the error, like it is formally proven, without deformations, for the eigenvalues of $T_{n}(f), f(\theta)=2-2 \cos (\omega \theta), \omega \geq 2$ integer, and $g(\theta)=1$ (see [12]). The latter observation indicates that a more complete study of this 'deformed' periodicity has to be considered in the future.

We finally observe that remarkable numerical results for the eigenvalues of $\mathcal{P}_{n}(f, g)$, as reported in Figs. 11, 12 and 13, really answer in the positive to the question posed in the title of the paper. In fact, we obtain almost machine precision for

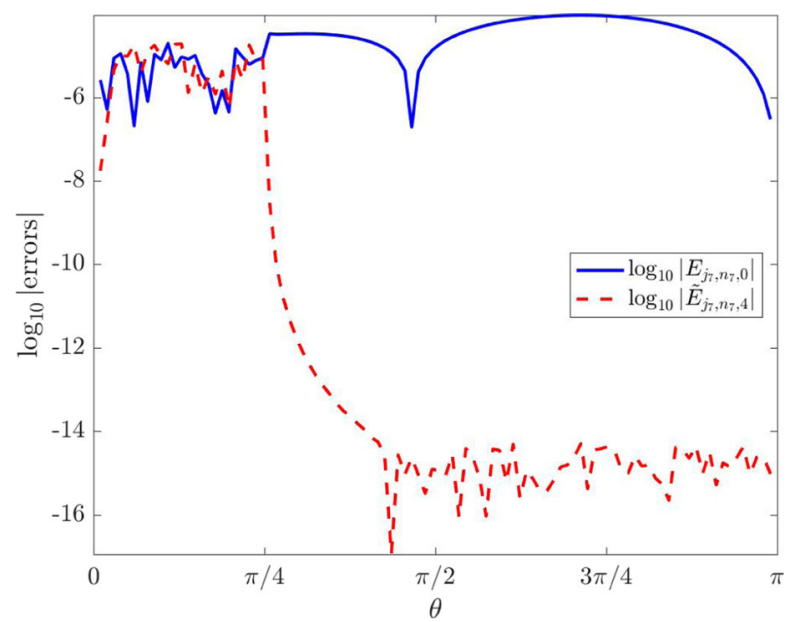

Fig. 13 Example 5: the errors $\log _{10}\left|E_{j_{7}, n_{7}, 0}\right|$ and $\log _{10}\left|\tilde{E}_{j_{7}, n_{7}, 4}\right|$ for the 100 indices $j_{7}$ of $n_{7}=6463$ in (18), corresponding to $n_{1}=100$, and using $\tilde{c}_{k}, k=1,2,3,4$, computed with $m=2$. Note the non monotone part, $\theta \in\left[0,2 \tan ^{-1}(\sqrt{3 / 17})\right]$, where the error is not improved 
the computation of the spectrum of $\mathcal{P}_{n}(f, g)$, for large $n$ and only working with few really small matrices.

\section{Conclusions}

Bogoya et al. [4-6] have recently obtained the precise asymptotic expansion for the eigenvalues of a sequence of Toeplitz matrices $\left\{T_{n}(f)\right\}$, under suitable assumptions on the associated generating function $f$. In this paper, we have shown numerical evidence that some of these assumptions can be relaxed and extended to the case of a sequence of preconditioned Toeplitz matrices $\left\{\mathcal{P}_{n}(f, g)=T_{n}^{-1}(g) T_{n}(f)\right\}$, for $f$ trigonometric polynomial, $g$ nonnegative, not identically zero trigonometric polynomial, $r=f / g$, and where the ratio $r$ plays the same role as $f$ in the nonpreconditioned case. The first-order asymptotic term of the expansion has been also proven using purely linear algebra tools.

Moreover, based on the eigenvalue asymptotics, we devised an extrapolation algorithm for computing the eigenvalues of preconditioned banded symmetric Toeplitz matrices with a high level of accuracy, with a relatively low computational cost, and with potential application to the computation of the spectrum of differential operators. In fact, up to low rank corrections, matrices of the form $\mathcal{P}_{n}(f, g)$ appear in the context of the spectral approximation of differential operators in which a low rank correction of $T_{n}(g)$ is the mass matrix and a low rank correction of $T_{n}(f)$ is the stiffness matrix. We carried out also preliminary numerical tests confirming that the same kind of asymptotic expansion holds, at least in the context of the Isogeometric approximation of second-order differential operators.

Therefore, a plan for the future has to include:

- the theoretical proof of the asymptotic expansion in (1) for $\alpha>1$;

- the analysis of the non monotone case and its relations with the study in [12] for the special case where $f(\theta)=2-2 \cos (\omega \theta), \omega \geq 2$ integer, and $g(\theta)=1$;

- the extension of the results by [1] to the preconditioned Toeplitz case and the study of its connection with the general expansion in (1);

- the extension of the numerical and theoretical study to a multidimensional, block setting, with special attention to the matrices coming from the approximation of elliptic differential operators.

Acknowledgements The research of Eman Salem Al-Aidarous was funded by King Abdulaziz University during scientific communication year 2017-2018. The research of Sven-Erik Ekström is cofinanced by the Graduate School in Mathematics and Computing (FMB) and Uppsala University. The research of the Isabella Furci and Stefano Serra-Capizzano is cofinanced by INdAM-GNCS (Istituto Nazionale di Alta Matematica - Gruppo Nazionale di Calcolo Scientifico).

Finally, a special thanks to the referee for pertinent comments, which helped us to improve the quality of the paper.

Open Access This article is distributed under the terms of the Creative Commons Attribution 4.0 International License (http://creativecommons.org/licenses/by/4.0/), which permits unrestricted use, distribution, and reproduction in any medium, provided you give appropriate credit to the original author(s) and the source, provide a link to the Creative Commons license, and indicate if changes were made. 


\section{Appendix}

Theorem 1 Let $f, g$ be real-valued cosine trigonometric polynomials (RCTP) on $[0, \pi]$ with $M_{g}=\max g>0$ and $m_{g}=\min g \geq 0$. If $r=\frac{f}{g}$ is monotone on $[0, \pi]$ then $\exists C>0$ such that

$$
\left|\lambda_{j}\left(\mathcal{P}_{n}(f, g)\right)-r\left(\frac{j \pi}{n+1}\right)\right| \leq C h \quad \forall j, \forall n,
$$

where

- $\mathcal{P}_{n}(f, g)$ is the "preconditioned" matrix $\mathcal{P}_{n}(f, g)=T_{n}^{-1}(g) T_{n}(f)$,

- $\lambda_{1}\left(\mathcal{P}_{n}(f, g)\right), \lambda_{2}\left(\mathcal{P}_{n}(f, g)\right), \ldots, \lambda_{n}\left(\mathcal{P}_{n}(f, g)\right)$ are the eigenvalues of $\mathcal{P}_{n}(f, g)$, arranged in nondecreasing or nonincreasing order, depending on whether $r$ is increasing or decreasing,

- $h=\frac{1}{n+1}$ and $\theta_{j, n}=\frac{j \pi}{n+1}=j \pi h$.

Proof For the sake of simplicity, we assume that $r$ is nondecreasing (the other case has a similar proof).

Notice that the conditions on $f$ and $g$ imply that $T_{n}(g)$ is positive definite and, by setting $\sim$ the symbol representing similarity between matrices, we find $\mathcal{P}_{n}(f, g) \sim$ $T_{n}^{-1 / 2}(g) T_{n}(f) T_{n}^{-1 / 2}(g)$ so we can order the eigenvalues of $\mathcal{P}_{n}(f, g)$ as follows

$$
\lambda_{1}\left(\mathcal{P}_{n}(f, g)\right) \leq \lambda_{2}\left(\mathcal{P}_{n}(f, g)\right) \leq \cdots \leq \lambda_{n}\left(\mathcal{P}_{n}(f, g)\right)
$$

We remark that

$$
\begin{aligned}
& T_{n}(f)=\tau_{n}(f)+H_{n}(f), \\
& T_{n}(g)=\tau_{n}(g)+H_{n}(g),
\end{aligned}
$$

where, for $\psi$ RCTP of degree $m$ and $Q=\left(\sqrt{\frac{2}{n+1}} \sin \left(\frac{i j \pi}{n+1}\right)\right)_{i, j=1}^{n}, \tau_{n}(\psi)$ is the following $\tau$ matrix [3] of size $n$ generated by $\psi$

$$
\tau_{n}(\psi)=Q \underset{1 \leq j \leq n}{\operatorname{diag}}\left(\psi\left(\frac{j \pi}{n+1}\right)\right) Q, \quad Q=Q^{T}=Q^{-1},
$$

and $H_{n}(\psi)$ is the Hankel matrix

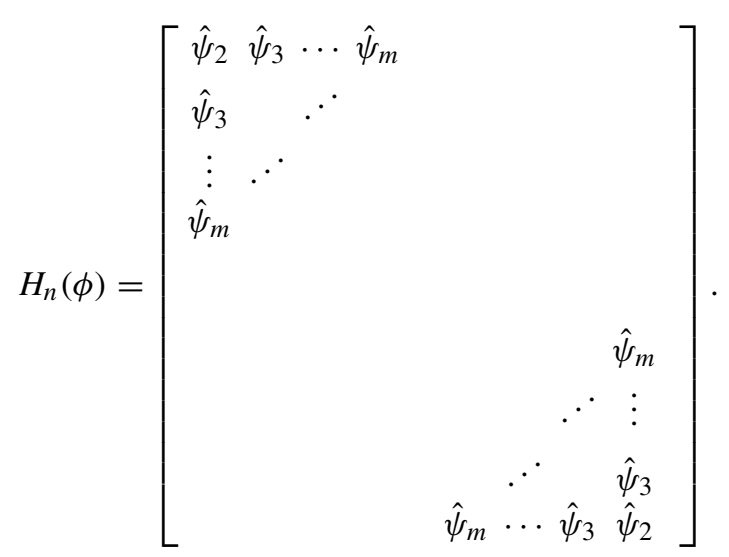


with $\operatorname{rank}\left(H_{n}(\psi)\right) \leq 2(m-1)$.

Hence,

$$
\begin{array}{cc}
R_{f} & :=\operatorname{rank}\left(H_{n}(f)\right) \leq 2(\operatorname{deg}(f)-1), \\
R_{g} & :=\operatorname{rank}\left(H_{n}(g)\right) \leq 2(\operatorname{deg}(g)-1), \\
R_{f, g}:=\max \left\{R_{f}, R_{g}\right\} \leq 2(\max \{\operatorname{deg}(f), \operatorname{deg}(g)\}-1) .
\end{array}
$$

Let $P_{n}^{\tau}$ be the matrix $\tau_{n}^{-1}(g) \tau_{n}(f)$,

$$
\begin{gathered}
P_{n}^{\tau}=Q\left(\underset{1 \leq j \leq n}{\operatorname{diag}}\left(g\left(\frac{j \pi}{n+1}\right)\right)\right)^{-1} Q Q \underset{1 \leq j \leq n}{\operatorname{diag}}\left(f\left(\frac{j \pi}{n+1}\right)\right) Q \\
=Q \operatorname{diag}\left(\frac{f}{g}\left(\frac{j \pi}{n+1}\right)\right) Q \\
=Q \operatorname{diag}\left(r\left(\frac{j \pi}{n+1}\right)\right) Q .
\end{gathered}
$$

Hence, for $j=1, \ldots, n$

$$
\lambda_{j}\left(P_{n}^{\tau}\right)=r\left(\frac{j \pi}{n+1}\right) .
$$

By observing that $T_{n}^{-1}(g) T_{n}(f)$ is similar to $T_{n}^{-1 / 2}(g) T_{n}(f) T_{n}^{-1 / 2}(g)$, using the MinMax spectral characterization for Hermitian matrices [2], fixed $j \in\left\{R_{f, g}+\right.$ $\left.1, \ldots, n-R_{f, g}\right\}$ and $T \subset \mathbb{C}^{n}, \operatorname{dim}(T)=n+1-j$, we obtain

$$
\begin{aligned}
& \lambda_{j}\left(\mathcal{P}_{n}(f, g)\right) \\
& =\lambda_{j}\left(T_{n}^{-1}(g) T_{n}(f)\right) \\
& =\lambda_{j}\left(T_{n}^{-1 / 2}(g) T_{n}(f) T_{n}^{-1 / 2}(g)\right) \\
& =\max _{\operatorname{dim}(T)=n+1-j}\left(\min _{\substack{x \in T \\
x \neq 0}}\left(\frac{x^{*} T_{n}^{-1 / 2}(g) T_{n}(f) T_{n}^{-1 / 2}(g) x}{x^{*} x}\right)\right) \\
& =\max _{\operatorname{dim}(T)=n+1-j}(\min \underbrace{}_{\substack{x \in T, x \neq 0 \\
y=T_{n}^{-1 / 2}(g) x}}\left(\frac{y^{*} T_{n}(f) y}{y^{*} T_{n}(g) y}\right)) \\
& =\max _{\operatorname{dim}(\hat{T})=n+1-j}\left(\min _{\substack{y \in \hat{T} \\
y \neq 0}}\left(\frac{y^{*} T_{n}(f) y}{y^{*} T_{n}(g) y}\right)\right),
\end{aligned}
$$

because $T_{n}^{-1 / 2}(g)$ is a full rank matrix and, if $\operatorname{dim}(T)=n+1-j$, then $\hat{T}:=\{y$ : $\left.y=T_{n}^{-1 / 2}(g) x, x \neq \underline{0}, x \in T\right\}$ is a new vector space having the same dimension $n+1-j$ as $T$.

Let $F$ be the subspace of $\mathbb{C}^{n}$ generated by the union of the columns of matrices $H_{n}(f)$ and $H_{n}(g)$. Because of the particular structure of the columns of Hankel matrices $H_{n}(f)$ and $H_{n}(g)$, we deduce

$$
\operatorname{dim}(F)=\max \left\{\operatorname{rank}\left(H_{n}(g)\right), \operatorname{rank}\left(H_{n}(f)\right)\right\}=R_{f, g},
$$

so that

Let us define $W_{f, g}=\hat{T} \cap F^{\perp}$,

$$
\operatorname{dim}\left(F^{\perp}\right)=n-R_{f, g} .
$$

$$
\begin{array}{r}
n+1-j \geq \operatorname{dim}\left(W_{f, g}\right) \geq \max \left\{0, \operatorname{dim}(\hat{T})+\operatorname{dim}\left(F^{\perp}\right)-n\right\}=n+1-j \\
+n-R_{f, g}-n=n+1-\left(j+R_{f, g}\right),
\end{array}
$$


because $n+1-\left(j+R_{f, g}\right) \geq 1$ for $j \leq n-R_{f, g}$. The latter implies in particular that $W_{f, g} \neq \emptyset$. Thus, due to the orthogonality, $\forall y \neq \underline{0} \in W_{f, g}$, we find

$$
H_{n}(f) y=\underline{0}, \quad H_{n}(g) y=\underline{0},
$$

so that

Hence, from (23)

$$
y^{*} H_{n}(f) y=0, \quad y^{*} H_{n}(g) y=0 .
$$

$\lambda_{j}\left(\mathcal{P}_{n}(f, g)\right)$

$$
\begin{aligned}
& =\max _{\operatorname{dim}(\hat{T})=n+1-j}\left(\min _{\substack{y \in \hat{T}, y \neq \underline{0}}}\left(\frac{y^{*}\left(\tau_{n}(f)+H_{n}(f)\right) y}{y^{*}\left(\tau_{n}(g)+H_{n}(g)\right) y}\right)\right) \\
& \leq \max _{\operatorname{dim}(\hat{T})=n+1-j}\left(\min _{\substack{y \in W_{f, g} \\
y \neq 0}}\left(\frac{y^{*}\left(\tau_{n}(f)+H_{n}(f)\right) y}{y^{*}\left(\tau_{n}(g)+H_{n}(g)\right) y}\right)\right) \\
& =\max _{\operatorname{dim}(\hat{T})=n+1-j}\left(\min _{\substack{y \in W_{f, g} \\
y \neq 0}}\left(\frac{y^{*} \tau_{n}(f) y}{y^{*} \tau_{n}(g) y}\right)\right) \\
& =\max _{\substack{W_{f, g}=\hat{T} \cap F^{\perp} \\
\operatorname{dim}(\hat{T})=n+1-j}}\left(\min _{\substack{y \in W_{f, g} \\
y \neq 0}},\left(\frac{y^{*} \tau_{n}(f) y}{y^{*} \tau_{n}(g) y}\right)\right) \\
& \leq \max _{n+1-j \geq \operatorname{dim}\left(\hat{W}_{f, g}\right) \geq n+1-\left(j+R_{f, g}\right)}\left(\min _{\substack{y \in \hat{W}_{f, g} \\
y \neq 0}},\left(\frac{y^{*} \tau_{n}(f) y}{y^{*} \tau_{n}(g) y}\right)\right) \\
& =\max _{n+1-j \geq \operatorname{dim}(\hat{W}) \geq n+1-\left(j+R_{f, g}\right)}\left(\min _{\substack{y \in \hat{W}_{f, g} \\
y \neq 0 \\
x=\tau_{n}^{1 / 2}(g) y}}\left(\frac{x^{*} \tau_{n}^{-1 / 2}(g) \tau_{n}(f) \tau_{n}^{-1 / 2}(g) x}{x^{*} x}\right)\right) \\
& =\max \left\{\lambda_{j}\left(P_{n}^{\tau}\right), \lambda_{j+1}\left(P_{n}^{\tau}\right), \ldots, \lambda_{j+R_{f, g}}\left(P_{n}^{\tau}\right)\right\} \\
& =\lambda_{j+R_{f, g}}\left(P_{n}^{\tau}\right) \text {. }
\end{aligned}
$$

By fixing $j \in\left\{R_{f, g}+1, \ldots, n-R_{f, g}\right\}$ and $T \subset \mathbb{C}^{n}, \operatorname{dim}(T)=j$, analogously we obtain

$$
\begin{array}{r}
\lambda_{j}\left(\mathcal{P}_{n}(f, g)\right)=\min _{\operatorname{dim}(T)=j}\left(\max _{\substack{x \in T, 0 \\
x \neq \underline{0}}}\left(\frac{x^{*} T_{n}^{-1 / 2}(g) T_{n}(f) T_{n}^{-1 / 2}(g) x}{x^{*} x}\right)\right) \\
=\min _{\operatorname{dim}(T)=j}\left(\max _{\substack{x \in T, x \neq 0 \\
y=T_{n}^{-1 / 2}(g) x}}\left(\frac{y^{*} T_{n}(f) y}{y^{*} T_{n}(g) y}\right)\right) \\
=\min _{\operatorname{dim}(\hat{T})=j}\left(\max _{\substack{y \in \hat{T}, y \neq \underline{0}}}\left(\frac{y^{*} T_{n}(f) y}{y^{*} T_{n}(g) y}\right)\right) \\
=\min _{\operatorname{dim}(\hat{T})=j}\left(\max _{\substack{y \in \hat{T} \\
y \neq \underline{0}}}\left(\frac{y^{*}\left(\tau_{n}(f)+H_{n}(f)\right) y}{y^{*}\left(\tau_{n}(g)+H_{n}(g)\right) y}\right)\right) .
\end{array}
$$

Let us define $W_{f, g}=\hat{T} \cap F^{\perp}$,

$j \geq \operatorname{dim}\left(W_{f, g}\right) \geq \max \left\{0, \operatorname{dim}(\hat{T})+\operatorname{dim}\left(F^{\perp}\right)-n\right\}=j+n-R_{f, g}-n=j-R_{f, g}$, because $j-R_{f, g} \geq 1$ for $j \geq R_{f, g}+1$. The latter implies in particular that $W_{f, g} \neq \emptyset$, and hence, due to the orthogonality, $\forall y \neq \underline{0} \in W_{f, g}$, we have

$$
H_{n}(f) y=\underline{0}, \quad H_{n}(g) y=\underline{0},
$$


and therefore

$$
y^{*} H_{n}(f) y=0, \quad y^{*} H_{n}(g) y=0 .
$$

Thus, from (25)

$$
\begin{aligned}
& \lambda_{j}\left(\mathcal{P}_{n}(f, g)\right) \geq \min _{\operatorname{dim}(\hat{T})=j}\left(\max _{\substack{y \in W_{f, g} \\
y \neq 0}}\left(\frac{y^{*}\left(\tau_{n}(f)+H_{n}(f)\right) y}{y^{*}\left(\tau_{n}(g)+H_{n}(g)\right) y}\right)\right) \\
& =\min _{\operatorname{dim}(\hat{T})=j}\left(\max _{\substack{y \in W_{f, g}, y \neq \underline{0}}}\left(\frac{y^{*} \tau_{n}(f) y}{y^{*} \tau_{n}(g) y}\right)\right) \\
& =\min _{\substack{W_{f, g}=\hat{T} \cap F^{\perp} \\
\operatorname{dim}(\hat{T})=j}}\left(\max _{\substack{y \in W_{f, g} \\
x \neq \underline{g}}},\left(\frac{y^{*} \tau_{n}(f) y}{y^{*} \tau_{n}(g) y}\right)\right) \\
& \geq \min _{j \geq \operatorname{dim}\left(\hat{W}_{f, g}\right) \geq j-R_{f, g}}\left(\max _{\substack{y \in W_{f, g} \\
y \neq \underline{0}}},\left(\frac{y^{*} \tau_{n}(f) y}{y^{*} \tau_{n}(g) y}\right)\right) \\
& =\min \left\{\lambda_{j}\left(P_{n}^{\tau}\right), \lambda_{j-1}\left(P_{n}^{\tau}\right), \ldots, \lambda_{j-R_{f, g}}\left(P_{n}^{\tau}\right)\right\} \\
& =\lambda_{j-R_{f, g}}\left(P_{n}^{\tau}\right) \text {. }
\end{aligned}
$$

By exploiting the previous inequality, relations (22) and (24), we obtain for $j=$ $R_{f, g}+1, \ldots, n-R_{f, g}$

$$
r\left(\frac{(j-s) \pi}{n+1}\right)=\lambda_{j-s}\left(P_{n}^{\tau}\right) \leq \lambda_{j}\left(\mathcal{P}_{n}(f, g)\right) \leq \lambda_{j+s}\left(P_{n}^{\tau}\right)=r\left(\frac{(j+s) \pi}{n+1}\right),
$$

where $s=R_{f, g}$.

The function $r$ is a RCTP on $[0, \pi]$ and a monotone increasing function so we have, $\forall n$ and $\forall j=s+1, \ldots, n-s$,

$\lambda_{j}\left(\mathcal{P}_{n}(f, g)\right)-r\left(\frac{j \pi}{n+1}\right) \leq r\left(\frac{(j+s) \pi}{n+1}\right)-r\left(\frac{j \pi}{n+1}\right)=r^{\prime}(\bar{\theta}) \frac{s \pi}{n+1} \leq\left\|r^{\prime}\right\|_{\infty} s \pi h$,

with $\bar{\theta} \in\left(\frac{j \pi}{n+1}, \frac{(j+s) \pi}{n+1}\right)$ and

$$
\lambda_{j}\left(\mathcal{P}_{n}(f, g)\right)-r\left(\frac{j \pi}{n+1}\right) \geq r\left(\frac{(j-s) \pi}{n+1}\right)-r\left(\frac{j \pi}{n+1}\right) \geq-\left\|r^{\prime}\right\|_{\infty} s \pi h .
$$

By setting $C=\left\|r^{\prime}\right\|_{\infty} s \pi$, for $s+1 \leq j \leq n-s$, we obtain

$$
\left|\lambda_{j}\left(\mathcal{P}_{n}(f, g)\right)-r\left(\frac{j \pi}{n+1}\right)\right| \leq C h .
$$

Furthermore, from $[11] \forall j=1, \ldots, n$, we know that

$$
m_{r} \leq \lambda_{j}\left(\mathcal{P}_{n}(f, g)\right) \leq M_{r},
$$

where

$$
m_{r}=\min _{\theta \in[0, \pi]} r(\theta) ; \quad m_{r}=\max _{\theta \in[0, \pi]} r(\theta),
$$

with strict inequalities that is $m_{r}<\lambda_{j}\left(\mathcal{P}_{n}(f, g)\right)<M_{r}$ if $m_{r}<M_{r}$, while the case $m_{r}=M_{r}$ is in fact trivial. Hence, for $n-s<j \leq n$

$\left|r\left(\frac{j \pi}{n+1}\right)-\lambda_{j}\left(\mathcal{P}_{n}(f, g)\right)\right| \leq\left|r\left(\frac{j \pi}{n+1}\right)-r\left(\frac{n \pi}{n+1}\right)\right| \leq\left|r^{\prime}(\bar{\theta})\right|\left|\frac{(n-j) \pi}{n+1}\right|$, 
where $\bar{\theta} \in\left(\frac{j \pi}{n+1}, \frac{n \pi}{n+1}\right)$. If $n-s<j \leq n$ then $|n-j|<s$, so that

$$
\left|r\left(\frac{j \pi}{n+1}\right)-\lambda_{j}\left(\mathcal{P}_{n}(f, g)\right)\right| \leq\left\|r^{\prime}\right\|_{\infty} s \pi h=C h .
$$

For $1 \leq j<s+1$

$$
\left|r\left(\frac{j \pi}{n+1}\right)-\lambda_{j}\left(\mathcal{P}_{n}(f, g)\right)\right| \leq\left|r\left(\frac{j \pi}{n+1}\right)-r\left(\frac{\pi}{n+1}\right)\right| \leq\left|r^{\prime}(\bar{\theta})\right|\left|\frac{(j-1) \pi}{n+1}\right|,
$$

where $\bar{\theta} \in\left(\frac{\pi}{n+1}, \frac{j \pi}{n+1}\right)$. If $1 \leq j<s+1$ then $|j-1|<s$, so

$$
\left|r\left(\frac{j \pi}{n+1}\right)-\lambda_{j}\left(\mathcal{P}_{n}(f, g)\right)\right| \leq\left\|r^{\prime}\right\|_{\infty} s \pi h=C h .
$$

Hence,

$$
\left|\lambda_{j}\left(\mathcal{P}_{n}(f, g)\right)-r\left(\frac{j \pi}{n+1}\right)\right| \leq C h \quad \forall j \forall n .
$$

Here, we present a second proof of the previous theorem.

Proof We adopt the very same notation used for the first proof. First, we notice that the low rank matrices $H_{n}(f)$ and $H_{n}(g)$ are also Hermitian matrices because $T_{n}(f)$, $T_{n}(g), \tau_{n}(f)$, and $\tau_{n}(g)$ are Hermitian matrices. Let $\mathbf{x}_{i}$ and $\lambda_{i}\left(\mathcal{P}_{n}(f, g)\right)$ be a pair eigenvector and eigenvalue of $\mathcal{P}_{n}(f, g)$. Then we can write

$$
\mathcal{P}_{n}(f, g) \mathbf{x}_{i}=\lambda_{i}\left(\mathcal{P}_{n}(f, g)\right) \mathbf{x}_{i} .
$$

By multiplying the previous equation from the left by the matrix $T_{n}(g)=\tau_{n}(g)+$ $H_{n}(g)$, we obtain

$$
\left(\tau_{n}(f)+H_{n}(f)\right) \mathbf{x}_{i}=\lambda_{i}\left(\mathcal{P}_{n}(f, g)\right)\left(\tau_{n}(g)+H_{n}(g)\right) \mathbf{x}_{i},
$$

which is equivalent to

$$
\left(\tau_{n}(f)+H_{n}(f)-\lambda_{i}\left(\mathcal{P}_{n}(f, g)\right) H_{n}(g)\right) \mathbf{x}_{i}=\lambda_{i}\left(\mathcal{P}_{n}(f, g)\right) \tau_{n}(g) \mathbf{x}_{i}
$$

Finally, by setting $\mathbf{y}_{i}=\tau_{n}^{1 / 2}(g) \mathbf{x}_{i}$ and by multiplying from the left by the matrix $\tau_{n}^{-1 / 2}(g)$, we have

$$
\tau^{-1 / 2}(g)\left(\tau_{n}(f)+H_{n}(f)-\lambda_{i}\left(\mathcal{P}_{n}(f, g)\right) H_{n}(g)\right) \tau^{-1 / 2}(g) \mathbf{y}_{i}=\lambda_{i}\left(\mathcal{P}_{n}(f, g)\right) \mathbf{y}_{i} .
$$

Equation (31) tells us that $\lambda_{i}\left(\mathcal{P}_{n}(f, g)\right)$ is also the eigenvalue of

$$
\tau_{n}^{-1 / 2}(g)\left(\tau_{n}(f)+H_{n}(f)-\lambda_{i}\left(\mathcal{P}_{n}(f, g)\right) H_{n}(g)\right) \tau_{n}^{-1 / 2}(g) .
$$

We can write

$$
\tau_{n}^{-1 / 2}(g)\left(\tau_{n}(f)+H_{n}(f)-\lambda_{i}\left(\mathcal{P}_{n}(f, g)\right) H_{n}(g)\right) \tau_{n}^{-1 / 2}(g)
$$

as

$$
\begin{gathered}
\tau_{n}^{-1 / 2}(g) \tau_{n}(f) \tau_{n}^{-1 / 2}(g)+\tau_{n}^{-1 / 2}(g)\left(H_{n}(f)-\lambda_{i}\left(\mathcal{P}_{n}(f, g)\right) H_{n}(g)\right) \tau_{n}^{-1 / 2}(g) \\
=\tau_{n}(f / g)+\tau_{n}^{-1 / 2}(g)\left(H_{n}(f)-\lambda_{i}\left(\mathcal{P}_{n}(f, g)\right) H_{n}(g)\right) \tau_{n}^{-1 / 2}(g) .
\end{gathered}
$$


Notice that the rank of any linear combination of $H_{n}(f)$ and $H_{n}(g)$ is $R_{f, g}=$ $\max \left\{\operatorname{rank}\left(H_{n}(f)\right), \operatorname{rank}\left(H_{n}(g)\right)\right\}$ and the argument is the special Hankel structure of $H_{n}(f)$ and $H_{n}(g)$. As a conclusion, from the expression above, using the MinMax characterization and the interlacing theorem for Hermitian matrices, we write

$$
\lambda_{i-R_{f, g}}\left(\tau_{n}(f / g)\right) \leq \lambda_{i}\left(\mathcal{P}_{n}(f, g)\right) \leq \lambda_{i+R_{f, g}}\left(\tau_{n}(f / g)\right),
$$

where $i \in\left\{R_{f, g}-1, \cdots, n-R_{f, g}\right\}$, which leads again to the proof of Theorem 1 .

Remark With regard to Theorem 1, the case where $r$ is bounded and nonmonotone is even easier. If we consider $\hat{r}$, the monotone nondecreasing rearrangement of $r$ on $[0, \pi]$, taking into account that the derivative of $r$ has at most a finite number $S$ of sign changes, we deduce that $\hat{r}$ is Lipschitz continuous and its Lipschitz constant is bounded by $\left\|r^{\prime}\right\|_{\infty}$ (notice that $\hat{r}$ is not necessarily continuously differentiable, but the derivative of $\hat{r}$ has at most $S$ points of discontinuity). Furthermore, the eigenvalues of $\tau_{n}(r)$ are exactly given

$$
r\left(\frac{j \pi}{n+1}\right)
$$

so that, by ordering these values nondecreasingly, we deduce that they coincide with $\hat{r}\left(x_{j, h}\right)$, with $x_{j, h}$ of the form $\frac{j \pi}{n+1}(1+o(1))$. With these premises, the proof follows exactly the same steps as in Theorem 1, using the MinMax characterization and the interlacing theorem for Hermitian matrices.

\section{References}

1. Barrera, M., Grudsky, S.M.: Asymptotics of eigenvalues for pentadiagonal symmetric Toeplitz matrices. Oper. Theory Adv. Appl. 259, 51-77 (2017)

2. Bhatia, R.: Matrix Analysis Graduate Texts in Mathematics, vol. 169. Springer, New York (1997)

3. Bini, D., Capovani, M.: Spectral and computational properties of band symmetric Toeplitz matrices. Linear Algebra Appl. 52-53, 99-126 (1983)

4. Bogoya, J.M., Böttcher, A., Grudsky, S.M., Maximenko, E.A.: Eigenvalues of Hermitian Toeplitz matrices with smooth simple-loop symbols. J. Math. Anal. Appl. 422, 1308-1334 (2015)

5. Bogoya, J.M., Grudsky, S.M., Maximenko, E.A.: Eigenvalues of Hermitian Toeplitz matrices generated by simple-loop symbols with relaxed smoothness. Oper. Theory Adv. Appl. 259, 179-212 (2017)

6. Böttcher, A., Grudsky, S.M., Maximenko, E.A.: Inside the eigenvalues of certain Hermitian Toeplitz band matrices. J. Comput. Appl. Math. 233, 2245-2264 (2010)

7. Böttcher, A., Silbermann, B.: Introduction to Large Truncated Toeplitz Matrices. Springer (1999)

8. Brezinski, C., Redivo Zaglia, M.: Extrapolation Methods: Theory and Practice. Elsevier Science Publishers B.V, North-Holland (1991)

9. Chan, R.H., Ng, M.: Conjugate gradient methods for Toeplitz systems. SIAM Rev. 38-3, 427-482 (1996)

10. Chan, R.H., Tang, P.: Fast band-Toeplitz preconditioners for Hermitian Toeplitz systems. SIAM J. Sci. Comput. 15, 164-171 (1994)

11. Di Benedetto, F., Fiorentino, G., Serra, S.: C.G. Preconditioning for Toeplitz matrices. Comput. Math. Appl. 25-6, 33-45 (1993)

12. Ekström, S.-E., Serra-Capizzano, S.: Eigenvalues and Eigenvectors of Banded Toeplitz Matrices and the Related Symbols. Technical report, 2017-010, Department of Information Technology Uppsala University (2017) 
13. Ekström S.-E., Garoni C., Serra-Capizzano S.: Are the eigenvalues of banded symmetric Toeplitz matrices known in almost closed form? Exp. Math., in press (2017). https://doi.org/10.1080/10586458.2017.1320241

14. Garoni, C., Serra-Capizzano, S.: Generalized Locally Toeplitz Sequences: Theory and Applications, vol. I. Springer (2017)

15. Huckle, T., Serra-Capizzano, S., Tablino-Possio, C.: Preconditioning strategies for non-Hermitian Toeplitz linear systems. Numer. Linear Algebra Appl. 12-2/3, 211-220 (2005)

16. Huckle, T., Serra-Capizzano, S., Tablino-Possio, C.: Preconditioning strategies for Hermitian indefinite Toeplitz linear systems. SIAM. J. Sci. Comput. 25-5, 1633-1654 (2004)

17. Serra-Capizzano, S.: New PCG based algorithms for the solution of Hermitian Toeplitz systems. Calcolo 32, 53-176 (1995)

18. Serra-Capizzano, S.: Optimal, quasi-optimal and superlinear band-Toeplitz preconditioners for asymptotically ill-conditioned positive definite Toeplitz systems. Math. Comp. 66-218, 651-665 (1997)

19. Serra-Capizzano, S.: An ergodic theorem for classes of preconditioned matrices. Linear Algebra Appl. 282-1/3, 161-183 (1998)

20. Stoer, J., Bulirsch, R.: Introduction to Numerical Analysis, 3rd edn. Springer (2002) 